\title{
Cationic Palladium Complex Catalyzed Highly Enantioselective Intramolecular Addition of Arylboronic Acids to Ketones. A Convenient Synthesis of Optically Active Cycloalkanols
}

Guixia Liu and Xiyan Lu*

\section{State Key Laboratory of Organometallic Chemistry, Shanghai Institute of Organic Chemistry, Chinese Academy of Sciences, 354 Fenglin Lu, Shanghai 200032, China xylu@mail.sioc.ac.cn \\ Supporting Information (Part 2) \\ Table of Contents}

I. Copies of ${ }^{1} \mathrm{H},{ }^{13} \mathrm{C}$ and ${ }^{19} \mathrm{~F}$ NMR spectra (known compounds are not included)

1. Compound 3c

$\mathrm{S} 2-\mathrm{S} 3$

2. Compound 3d

S4-S6

3. Compound $3 \mathbf{e}$

S7-S8

4. Compound $\mathbf{3 f}$

S9-S10

5. Compound 3g

S11-S12

6. Compound $\mathbf{3 h}$

S13-S14

7. Compound $\mathbf{3 i}$

S15-S16

8. Compound $\mathbf{3 j}$

S17-S18

9. Compound 3k

S19-S20

10. Compound $\mathbf{2 b}$

S21-S22

11. Compound 2c

S23-S24

12. Compound 2d

S25-S27

13. Compound $2 \mathbf{e}$

S28-S29

14. Compound 2g

S30-S31

15. Compound $\mathbf{2 h}$

S32-S33

16. Compound $\mathbf{2 i}$

S34-S35

II. HPLC for the determination of ee values

1. Compound 2a

S36

2. Compound $\mathbf{2 b}$

S37

3. Compound 2c

S38

4. Compound 2d

S39

5. Compound $2 \mathbf{e}$

S40

6. Compound $2 \mathbf{f}$

S41

7. Compound $2 \mathrm{~g}$

S42

8. Compound $\mathbf{2 h}$

S43

9. Compound $\mathbf{2 i}$

S44

10. Compound $\mathbf{2} \mathbf{j}$

S45

11. Compound 2k

S46 


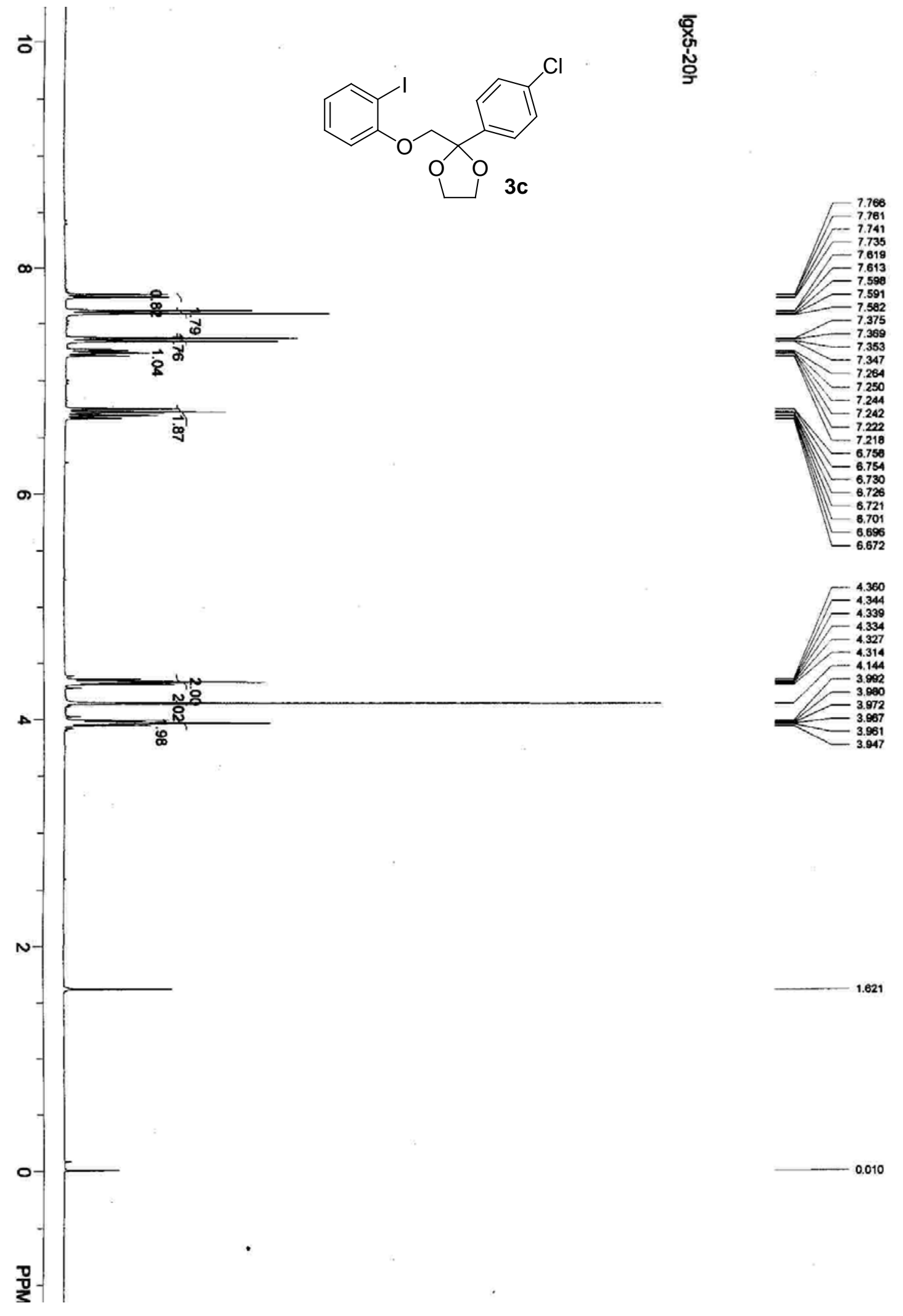



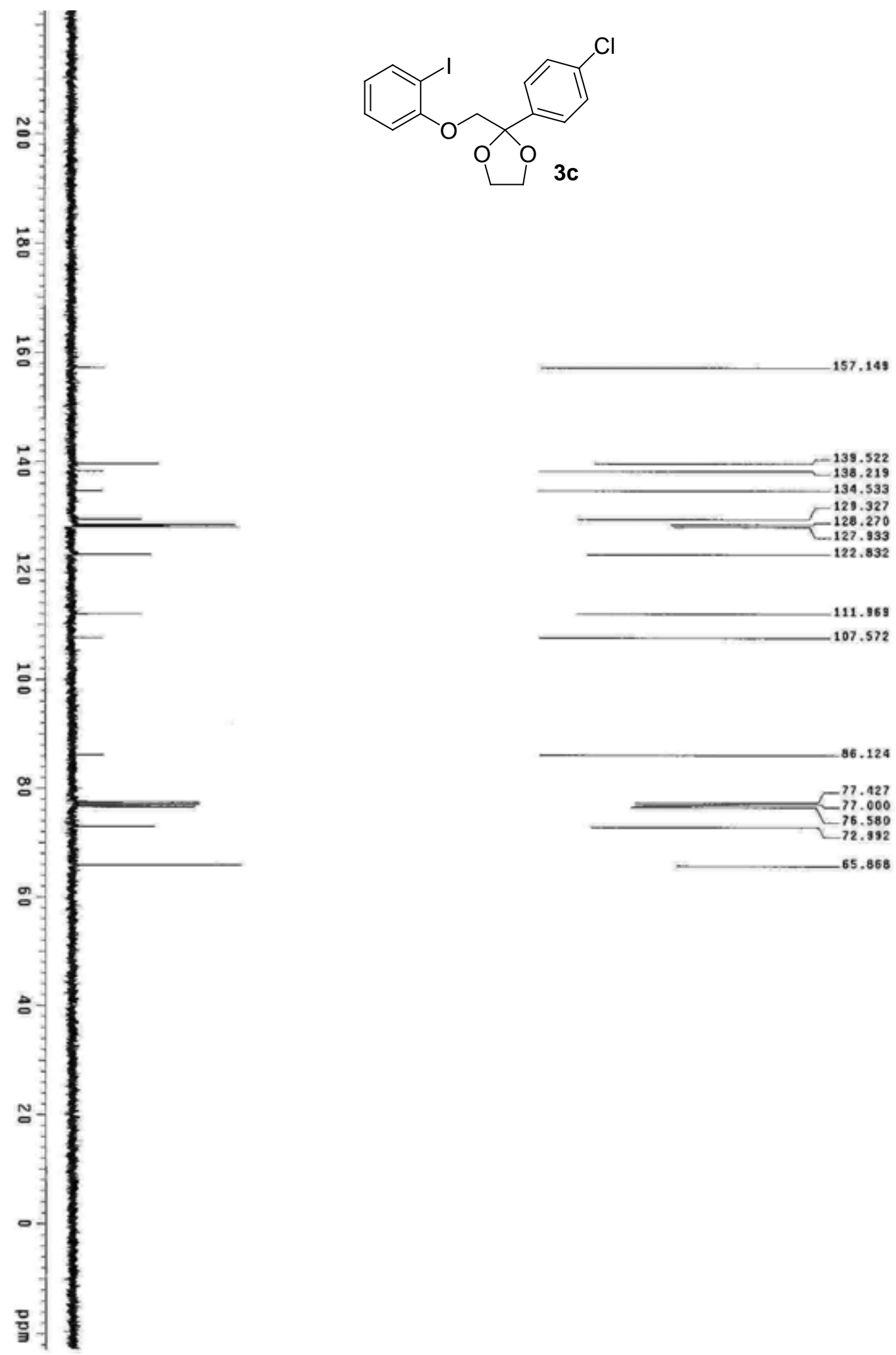


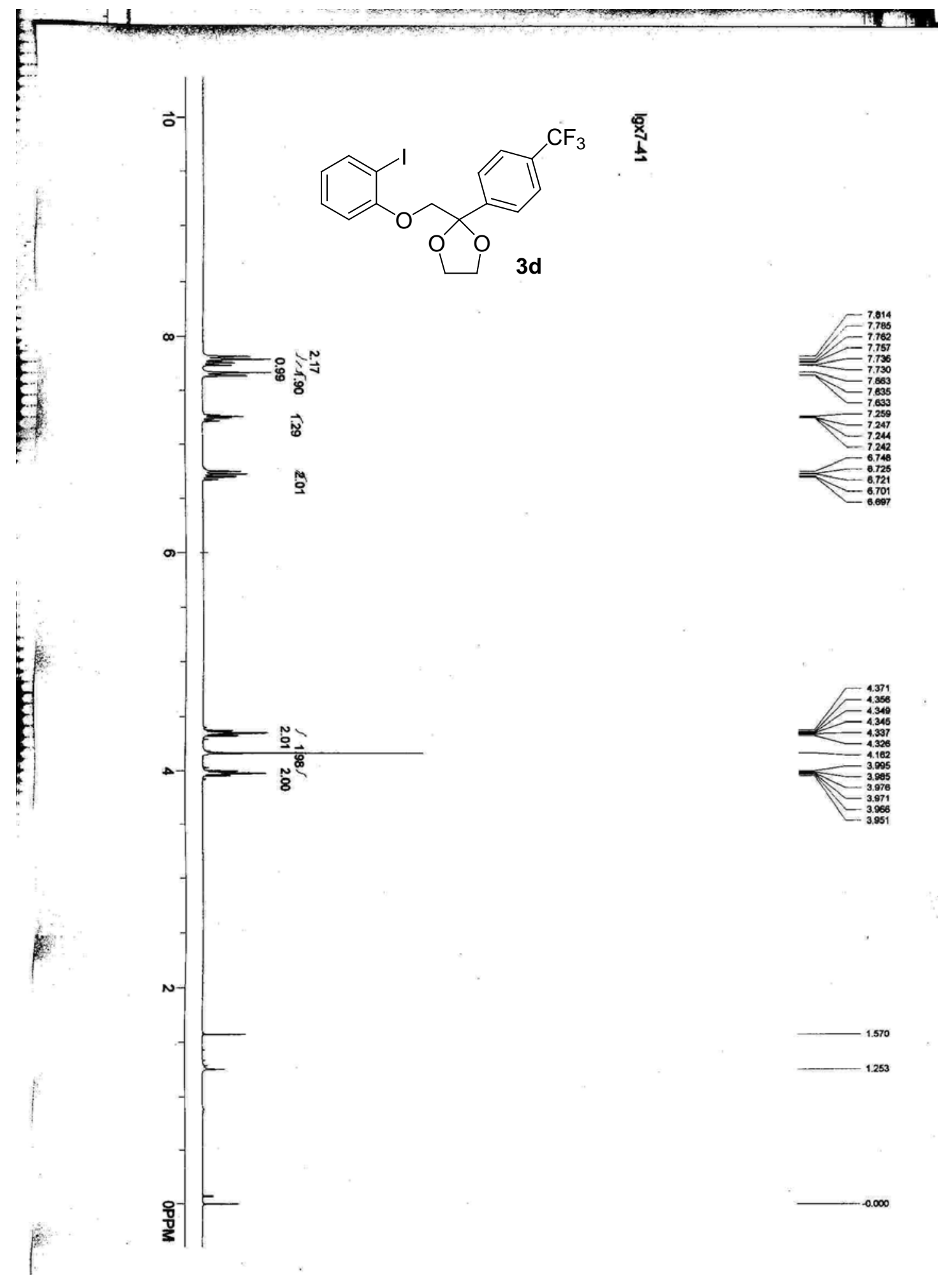




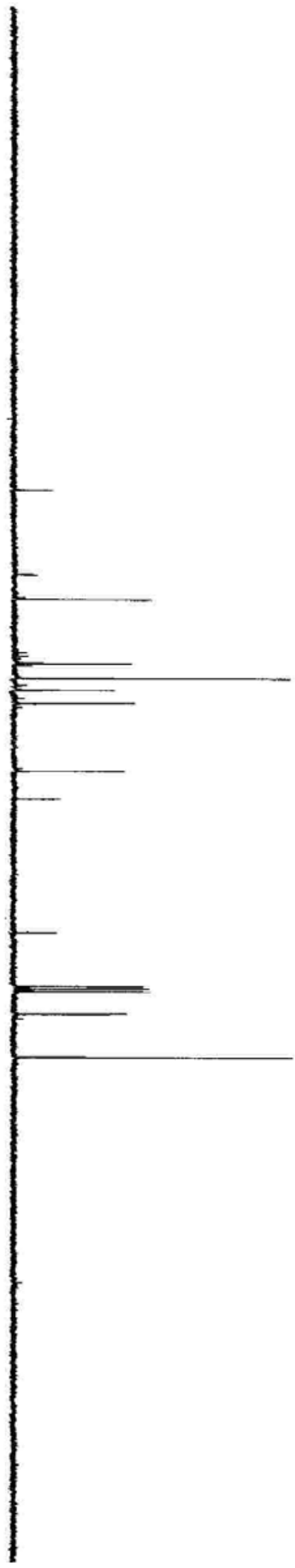

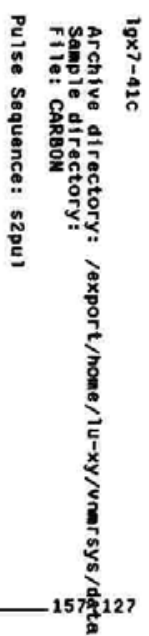

$-143.618$

$-139.587$

$-129.352$

$-125.162$

$-125.116$

125.063
-125.017

122.933

$-112.003$

$-102.538$

86.090

$-77.427$

77.000
76.580

76.580
-72.978

$-65.917$ 


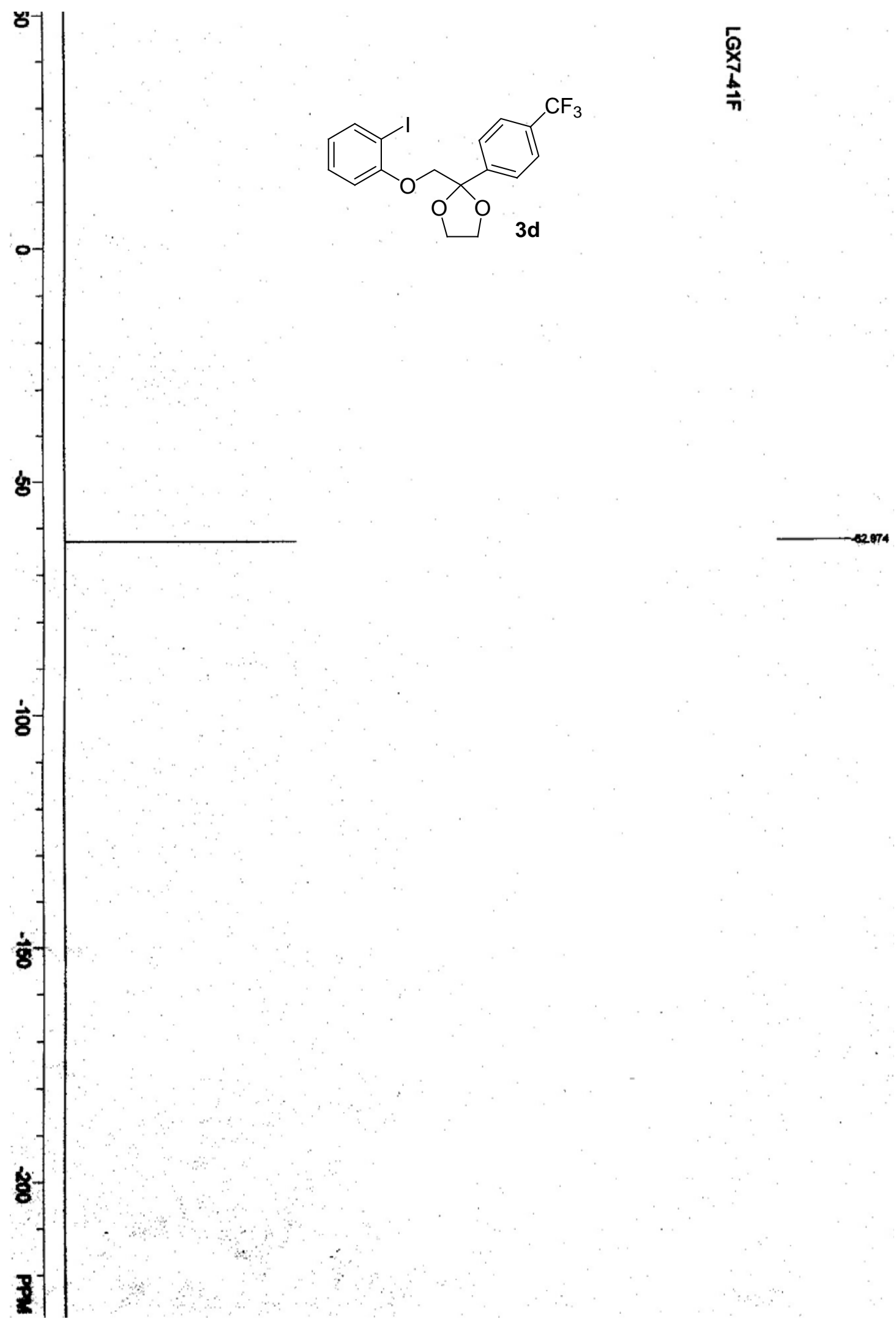




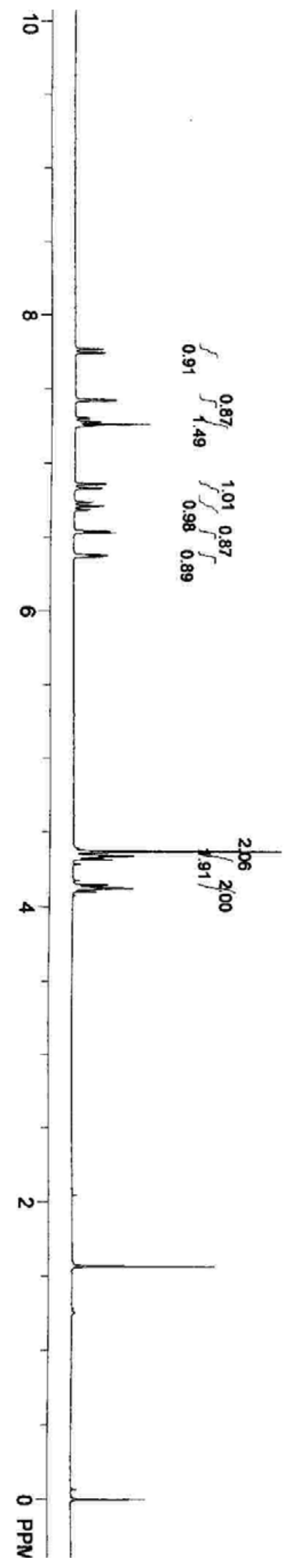

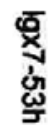
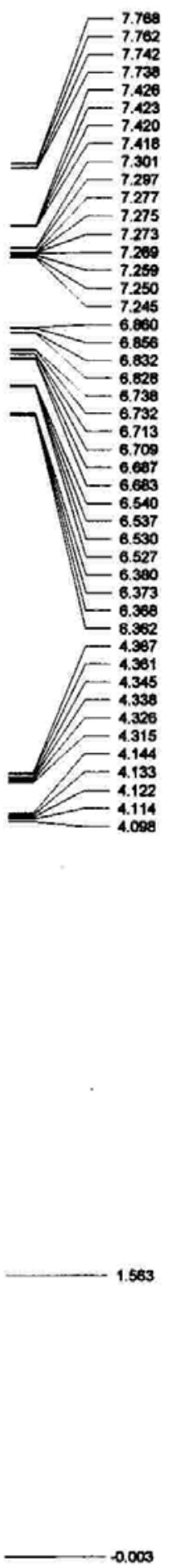


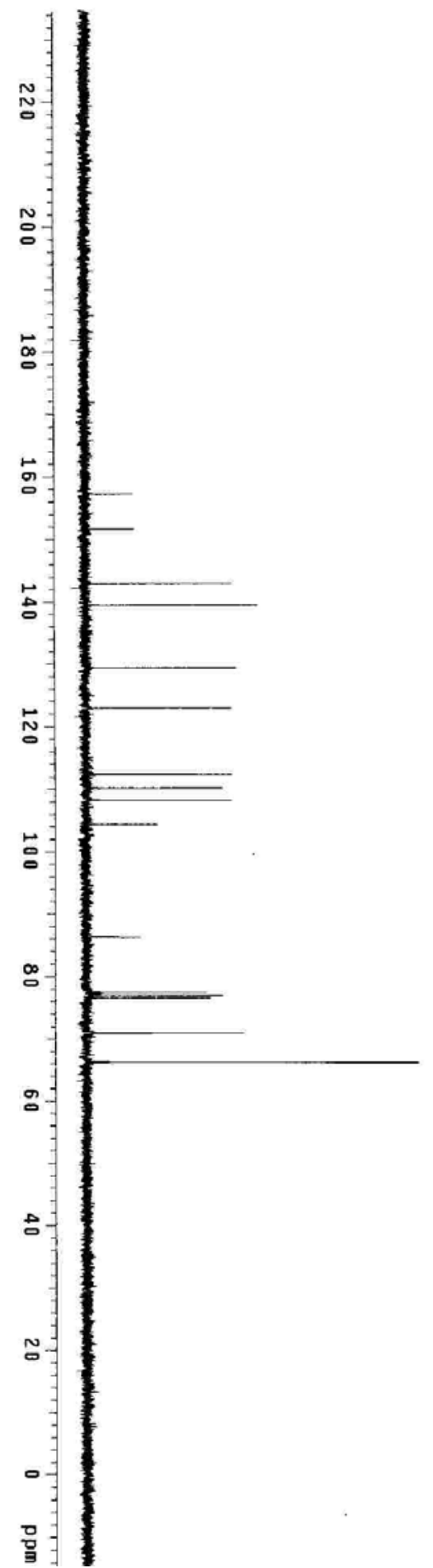

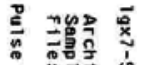

范

है

约

몰

$\square 3 e$

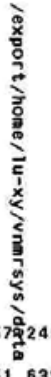



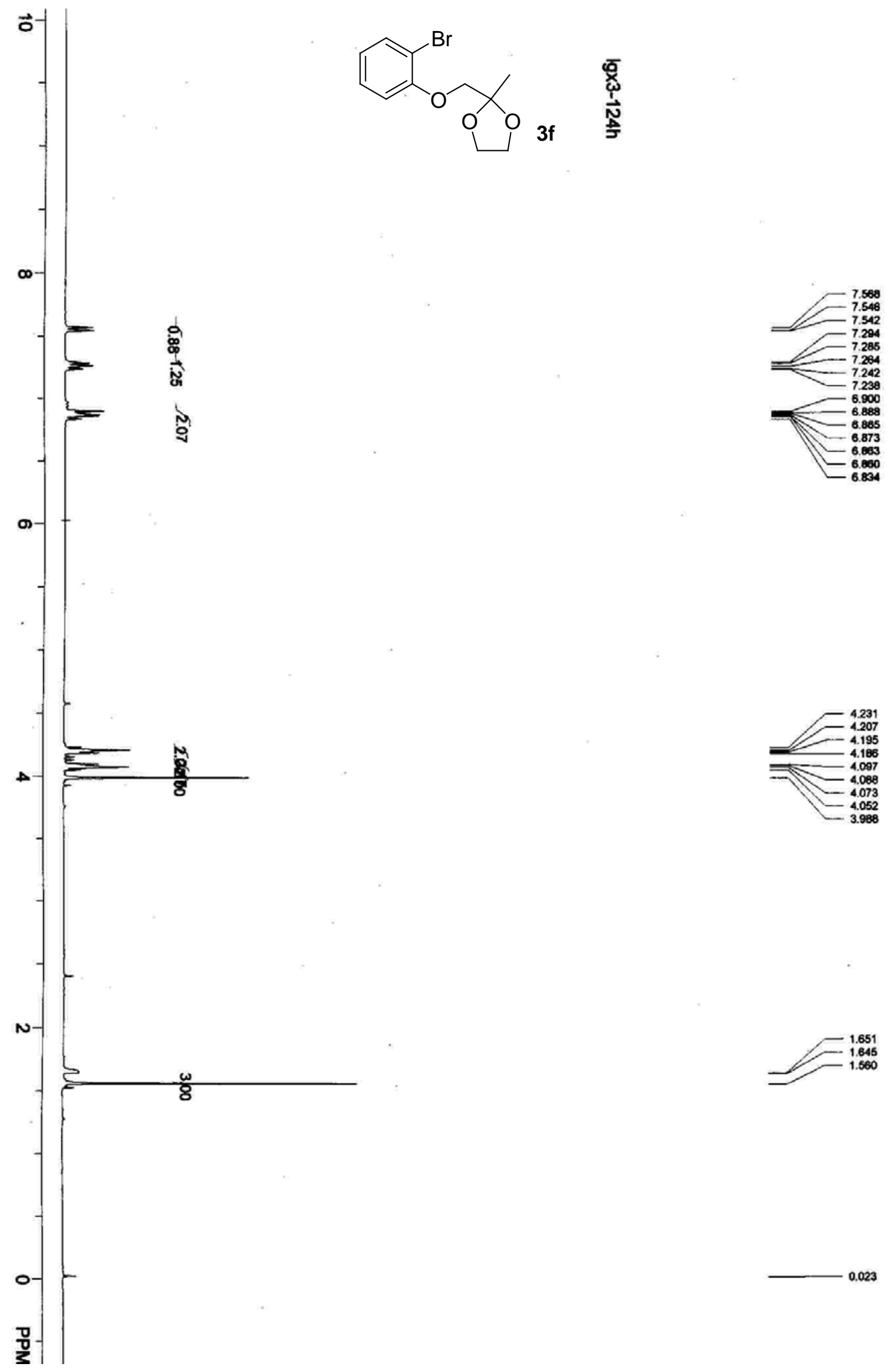

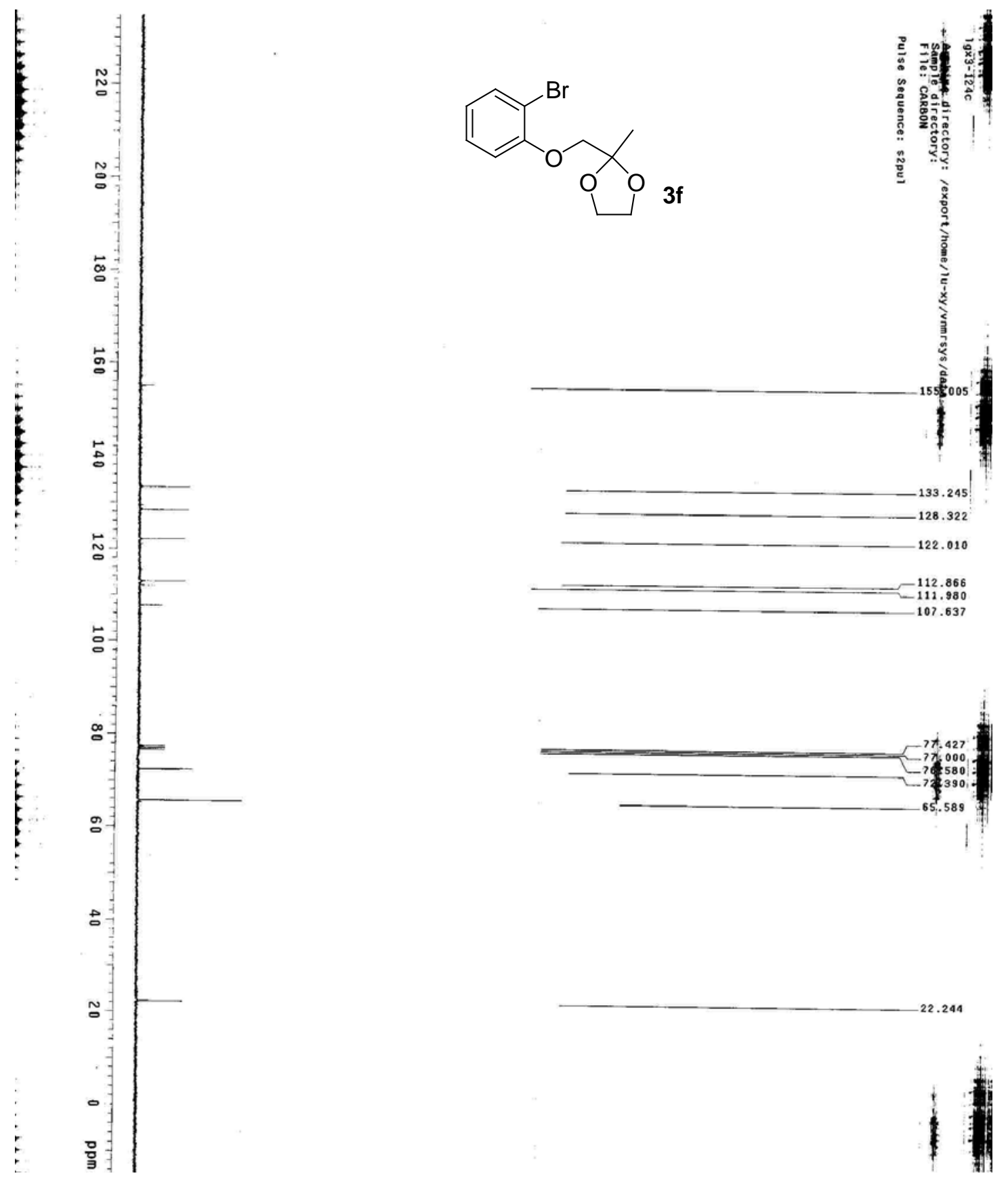


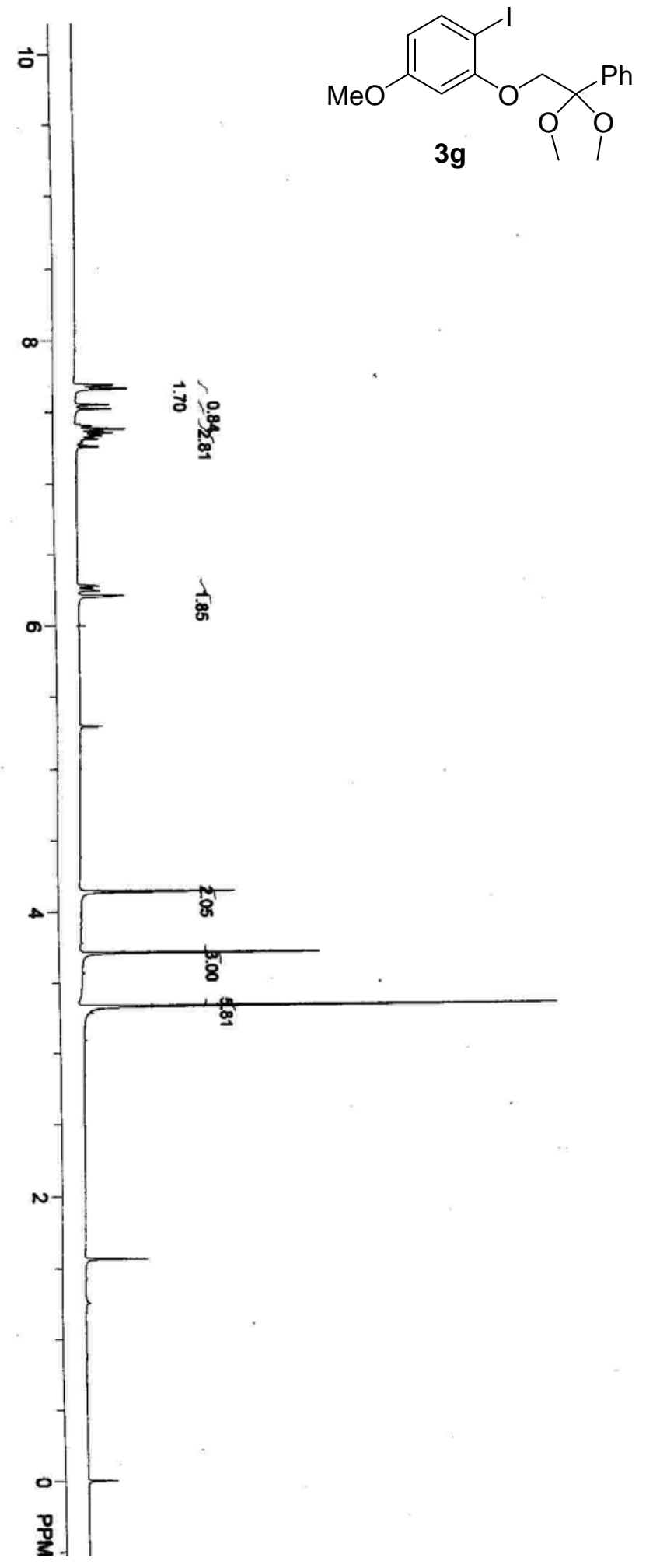

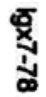
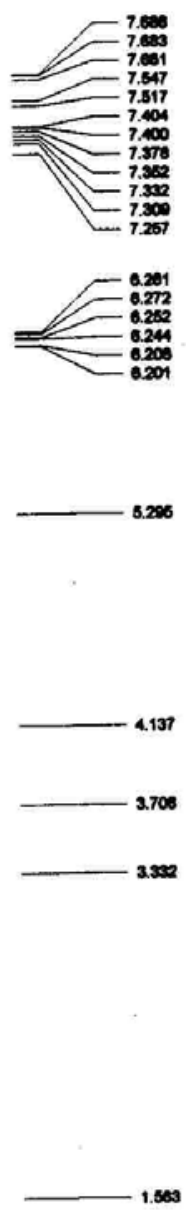

$-0.001$ 

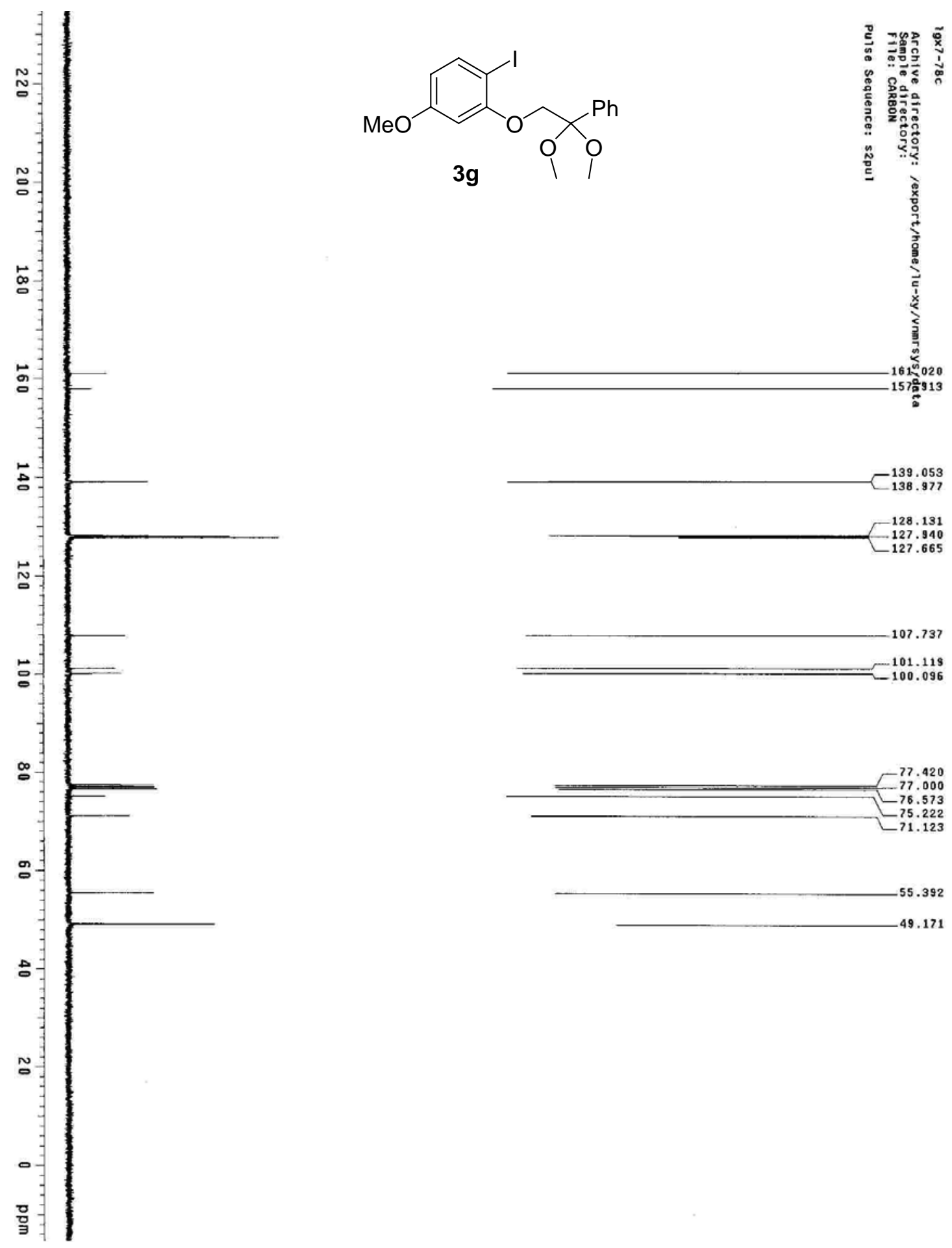


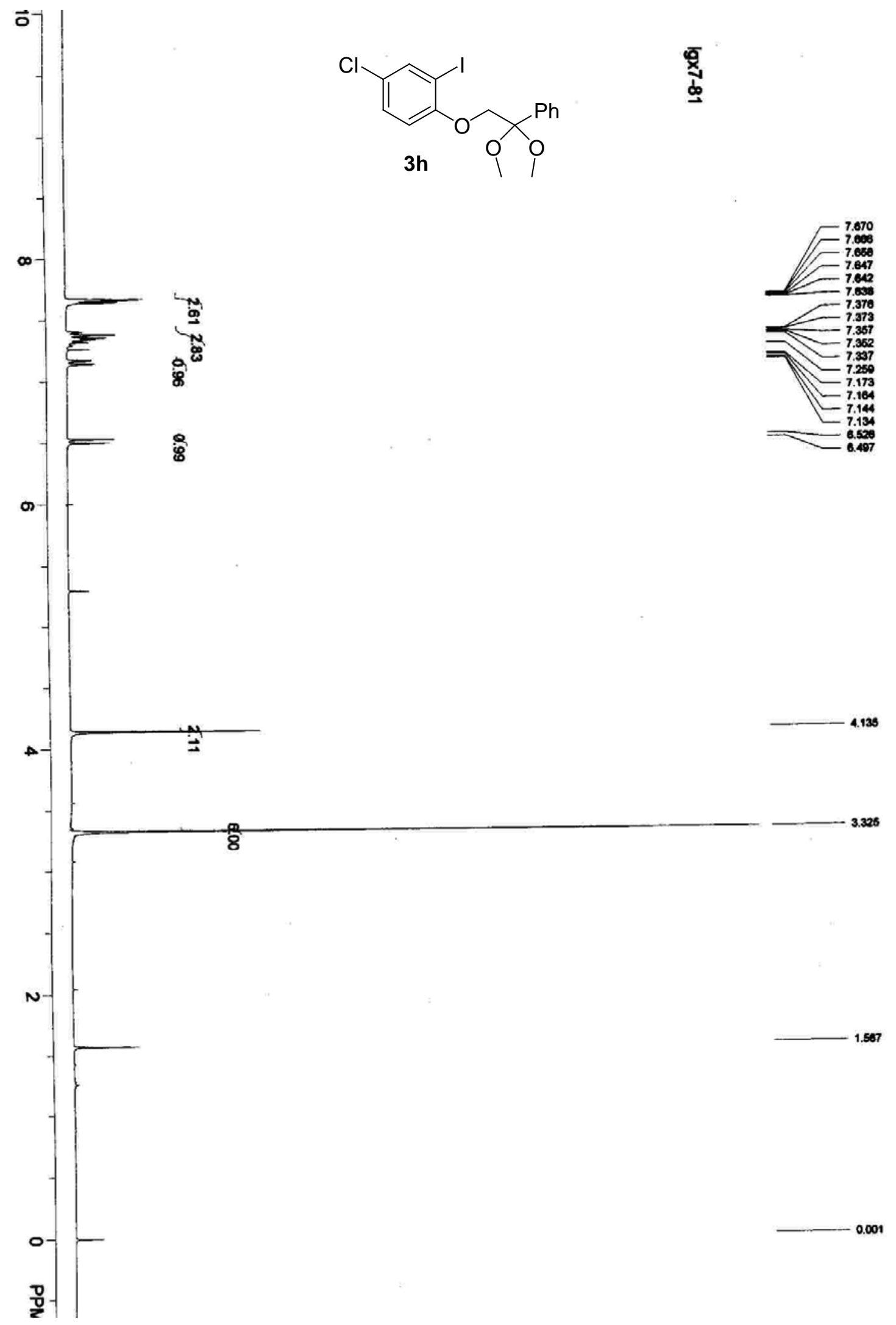



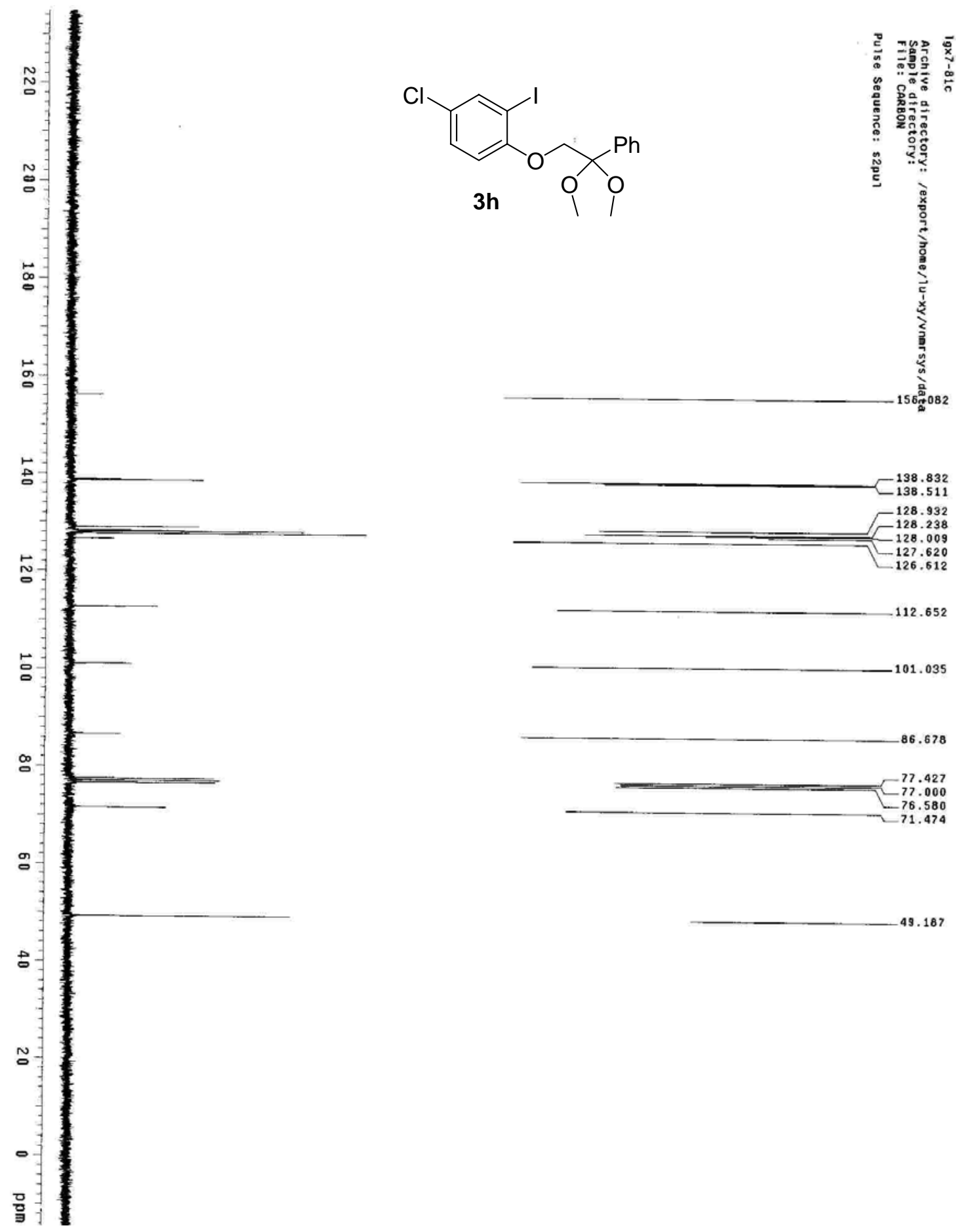

$-49.187$ 

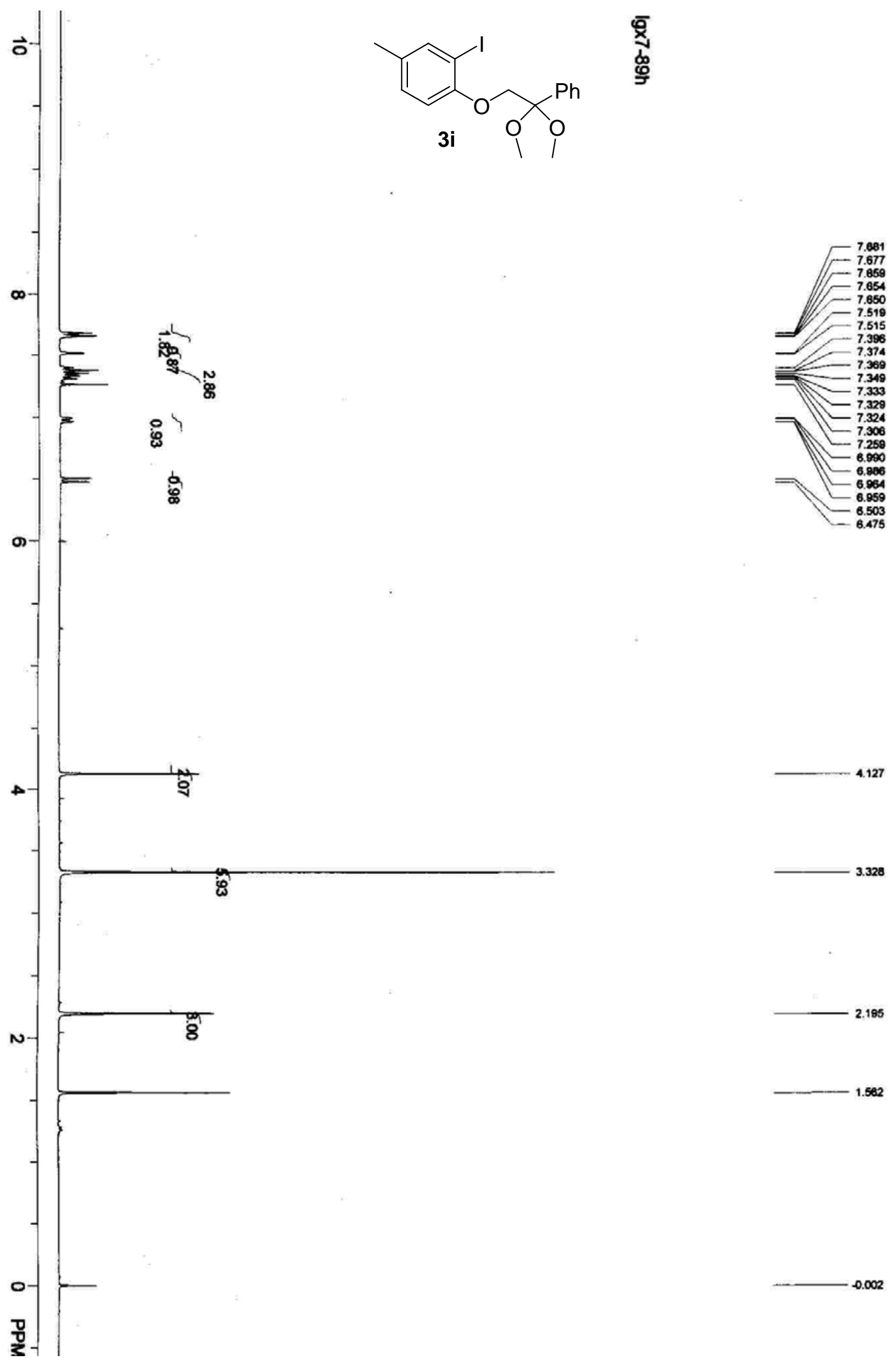

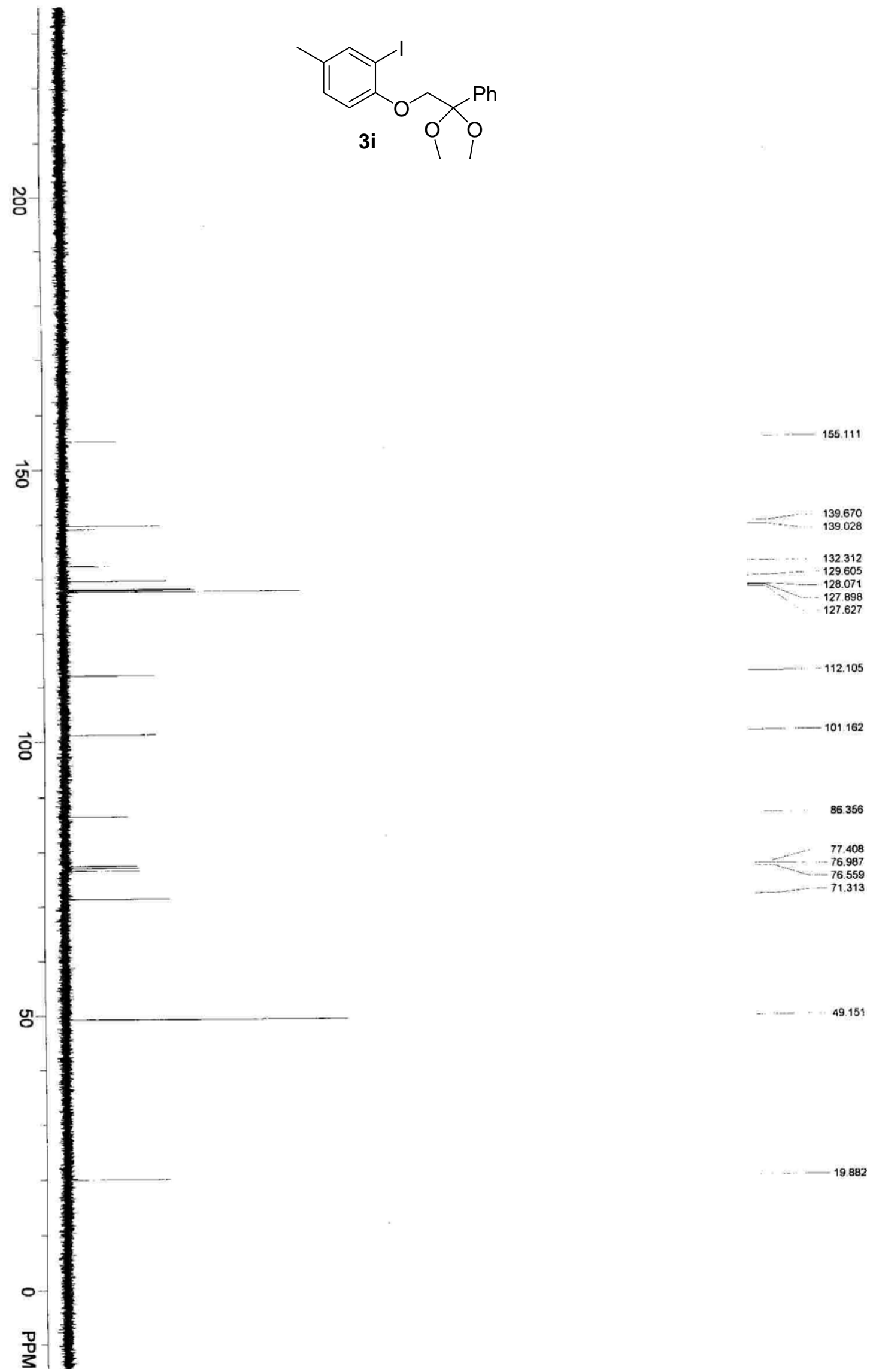


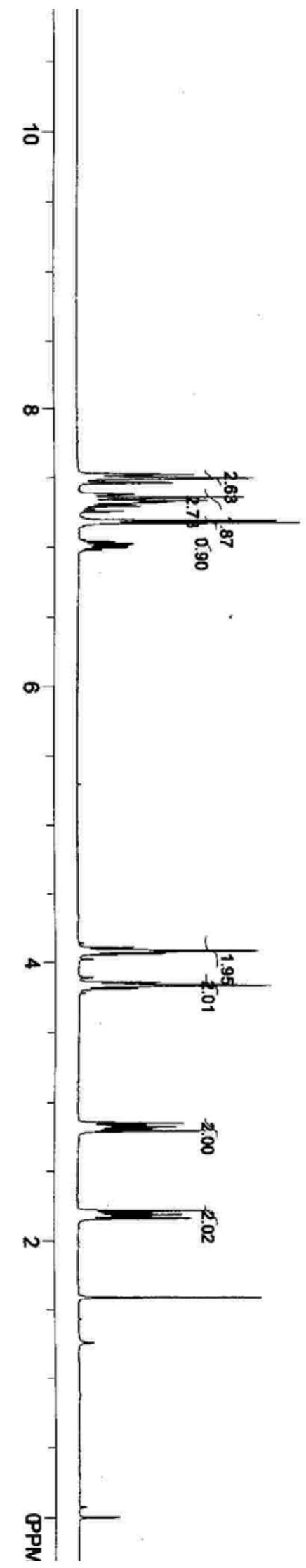

$\overline{\bar{x}}$
$\frac{\mathbf{x}}{\bar{\omega}}$
$\frac{\underline{y}}{y}$

3j
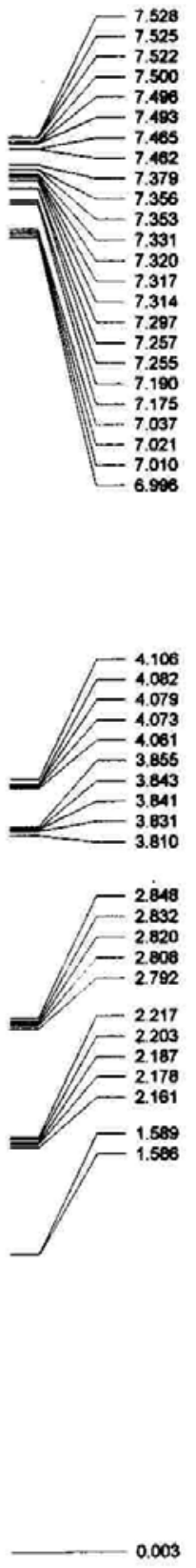

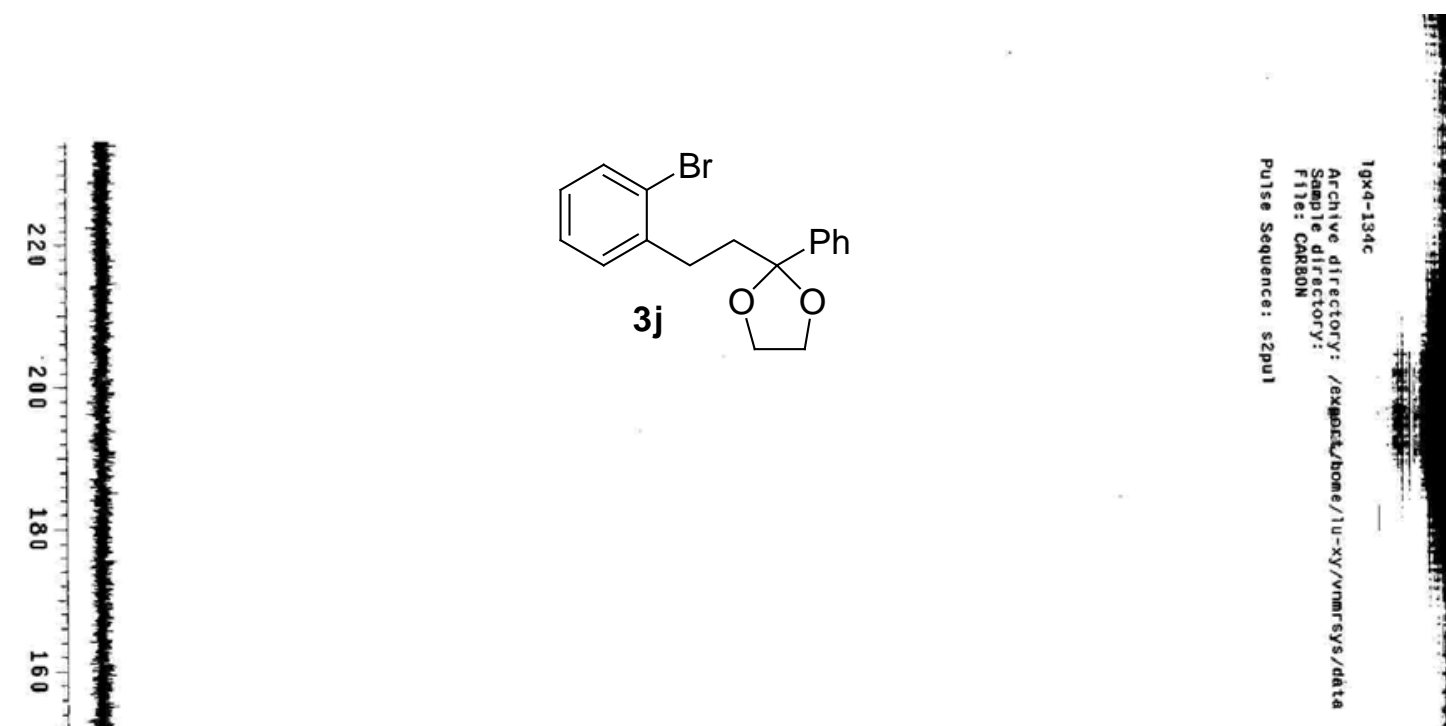

เ

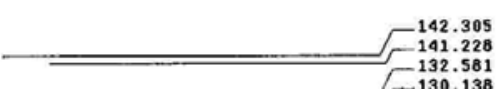

${ }^{132.581}$

$F_{-127.810}^{128.024}$

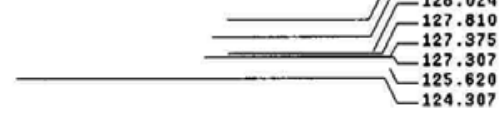

ㄴ.

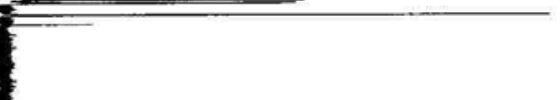

${ }_{-125.620}^{127}$

109.813

:

.

$\underbrace{71.043}_{717.048}$

$-64.544$

:-

$\therefore$

ㄱ.
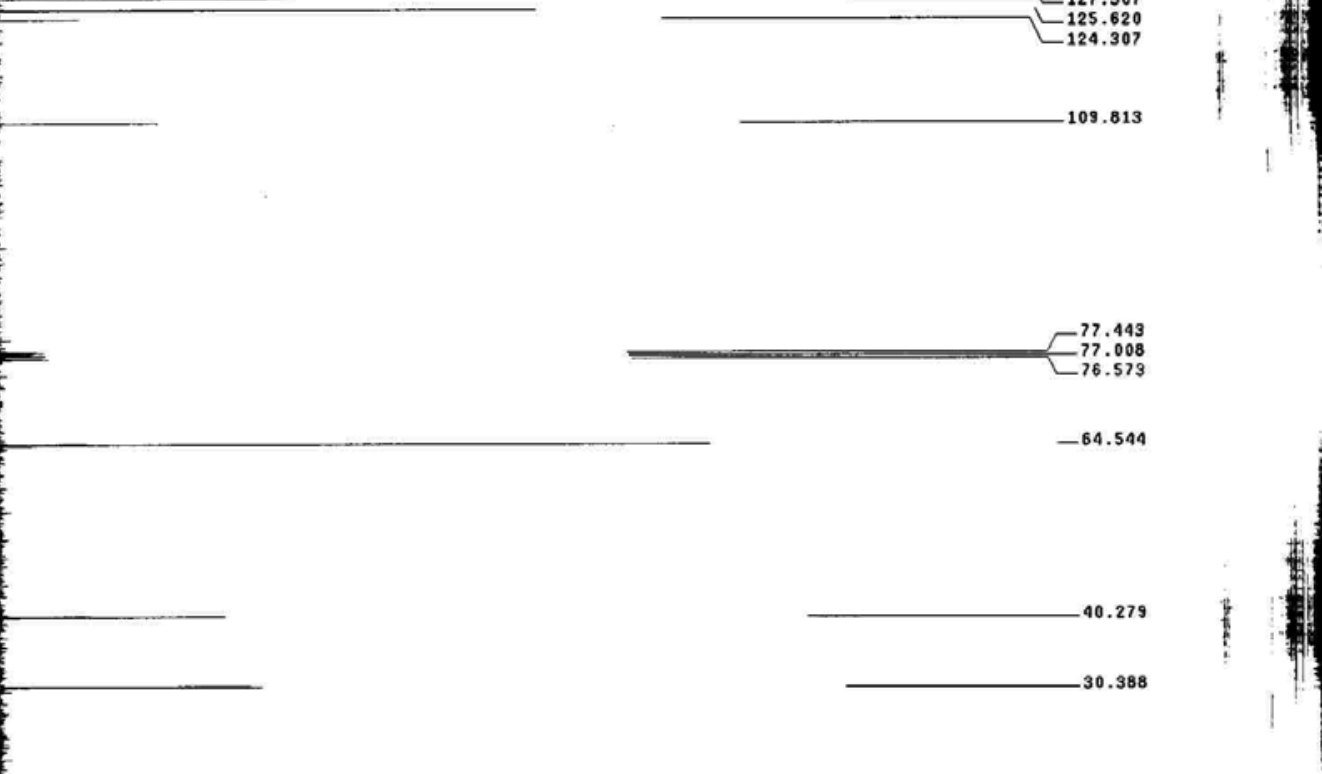

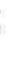



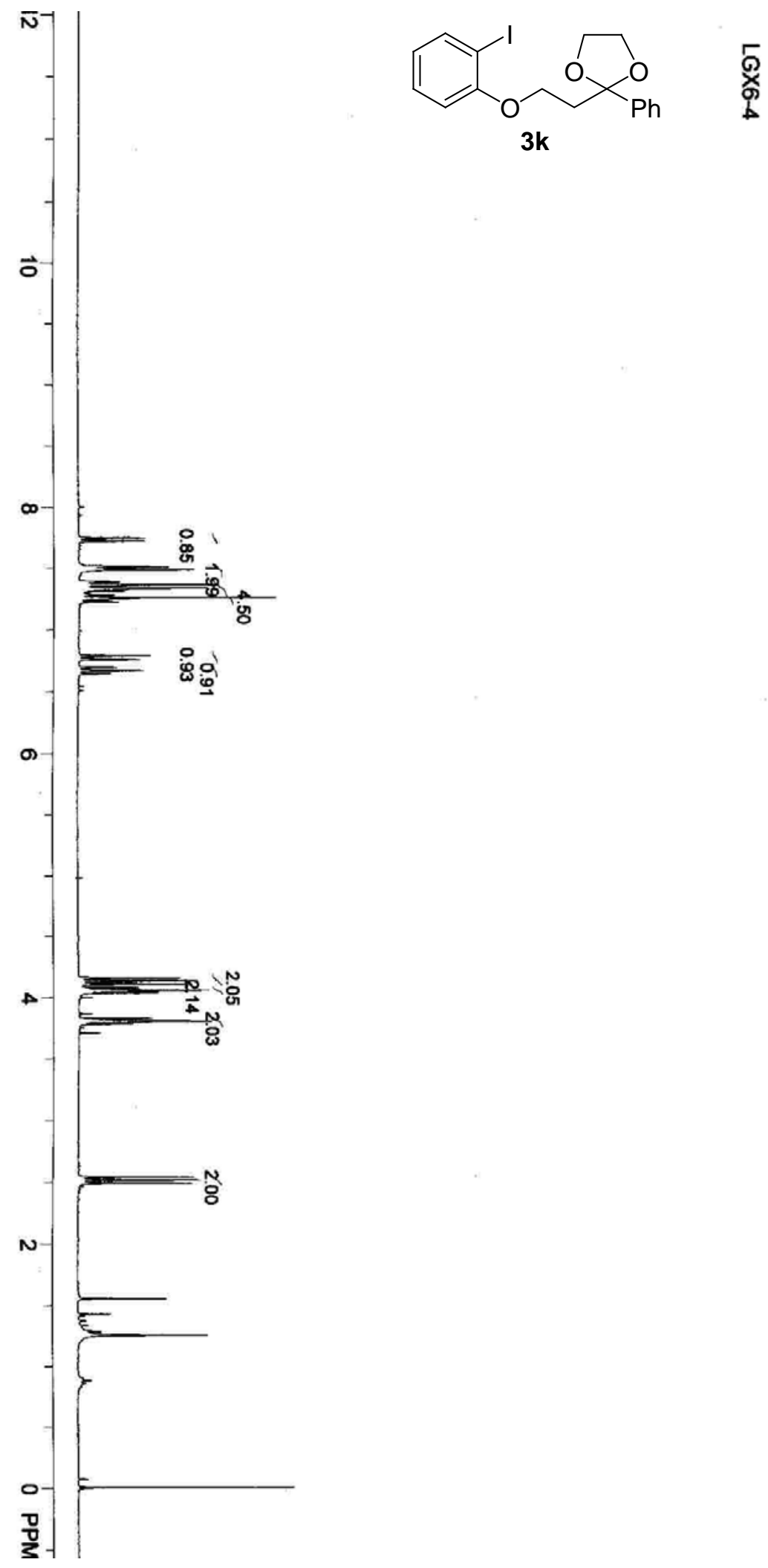

量
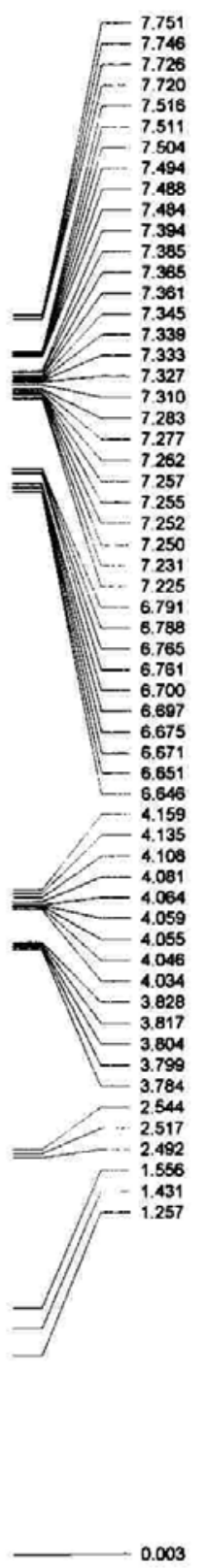

s- 


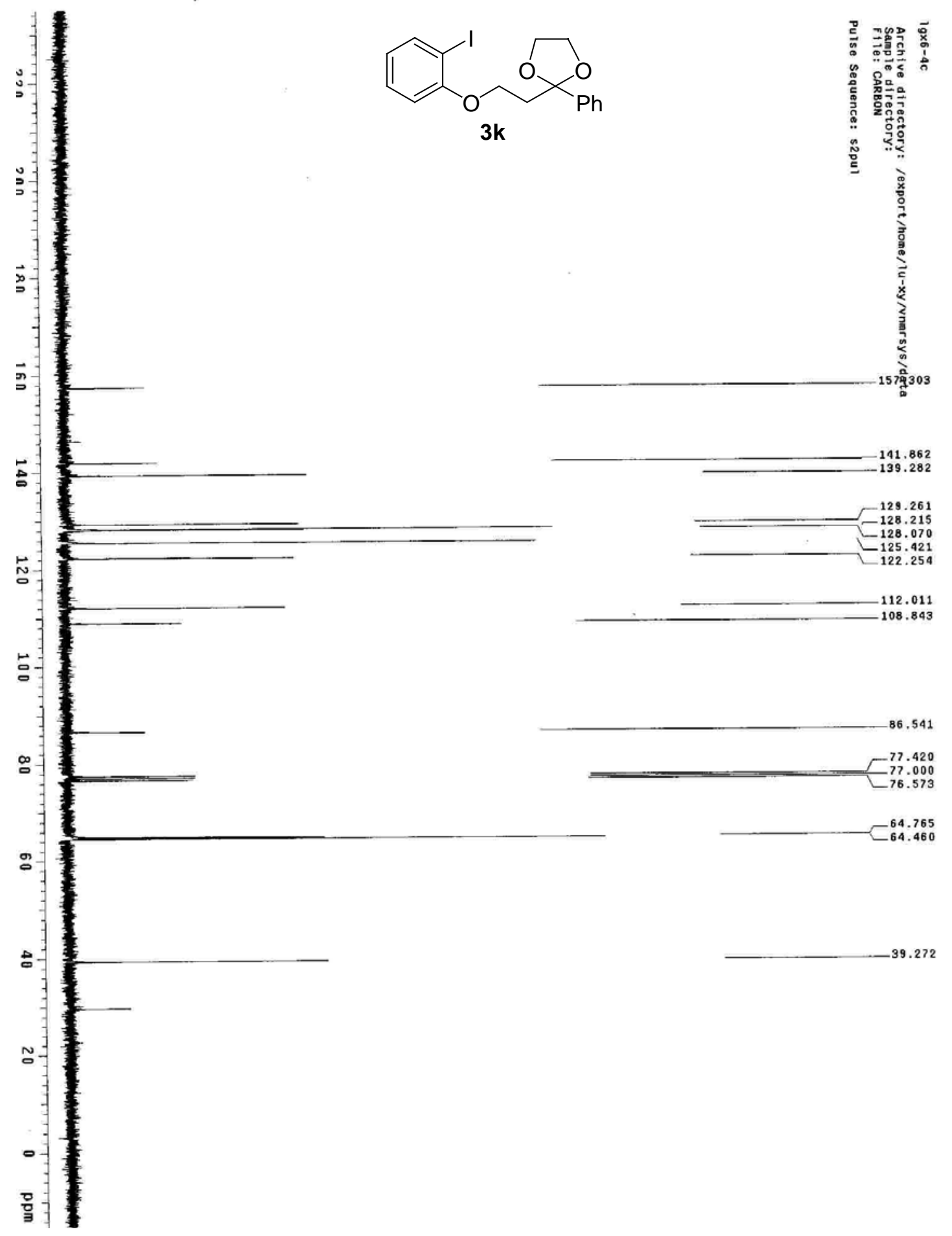




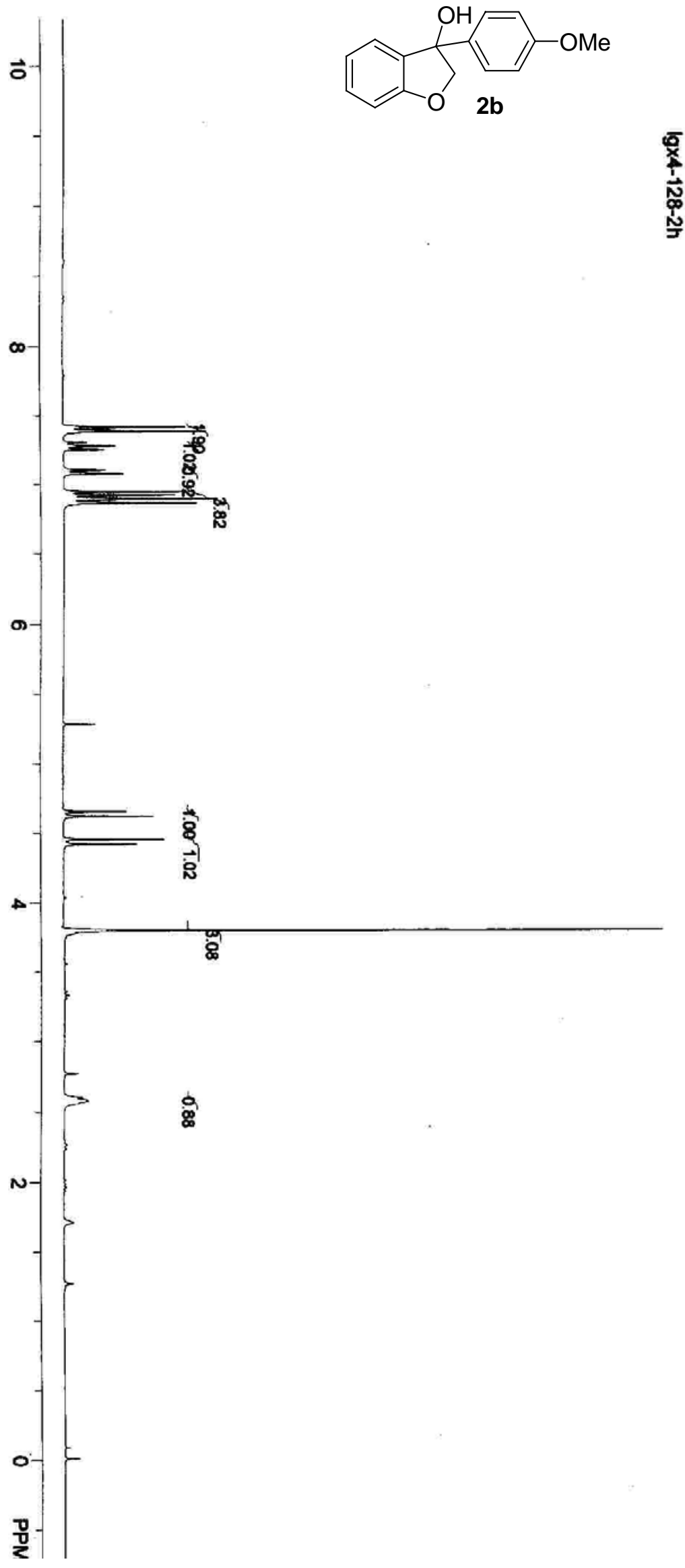

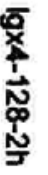
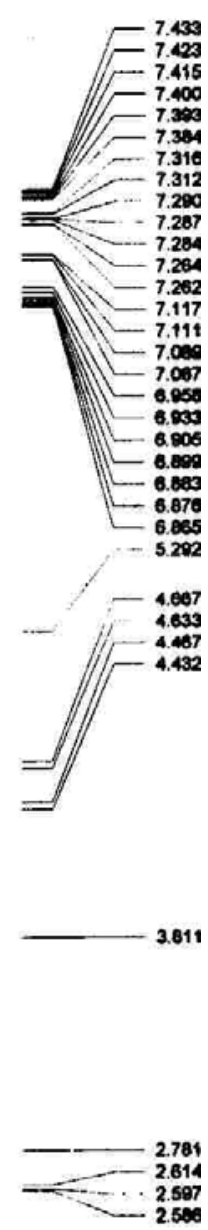

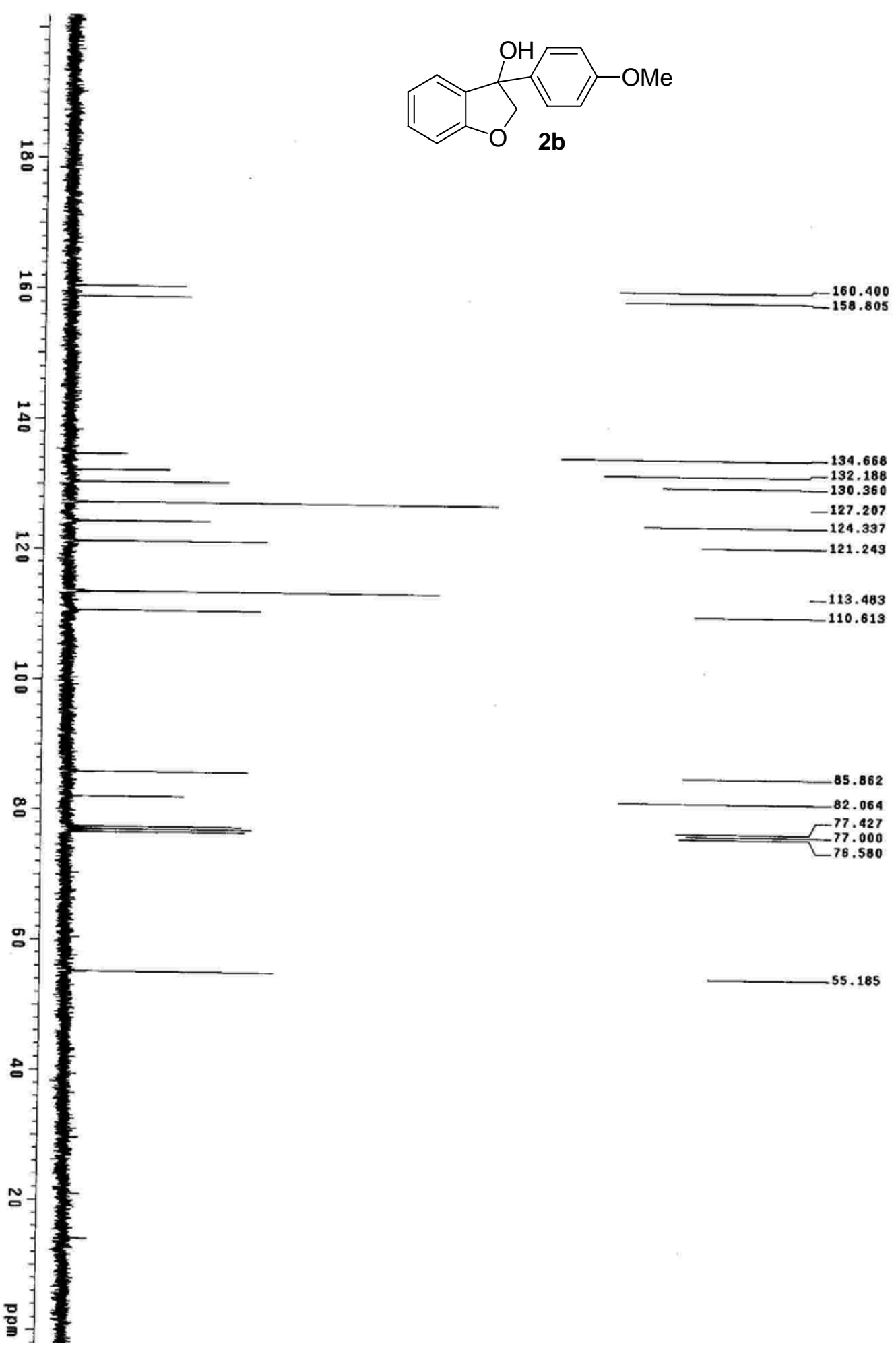


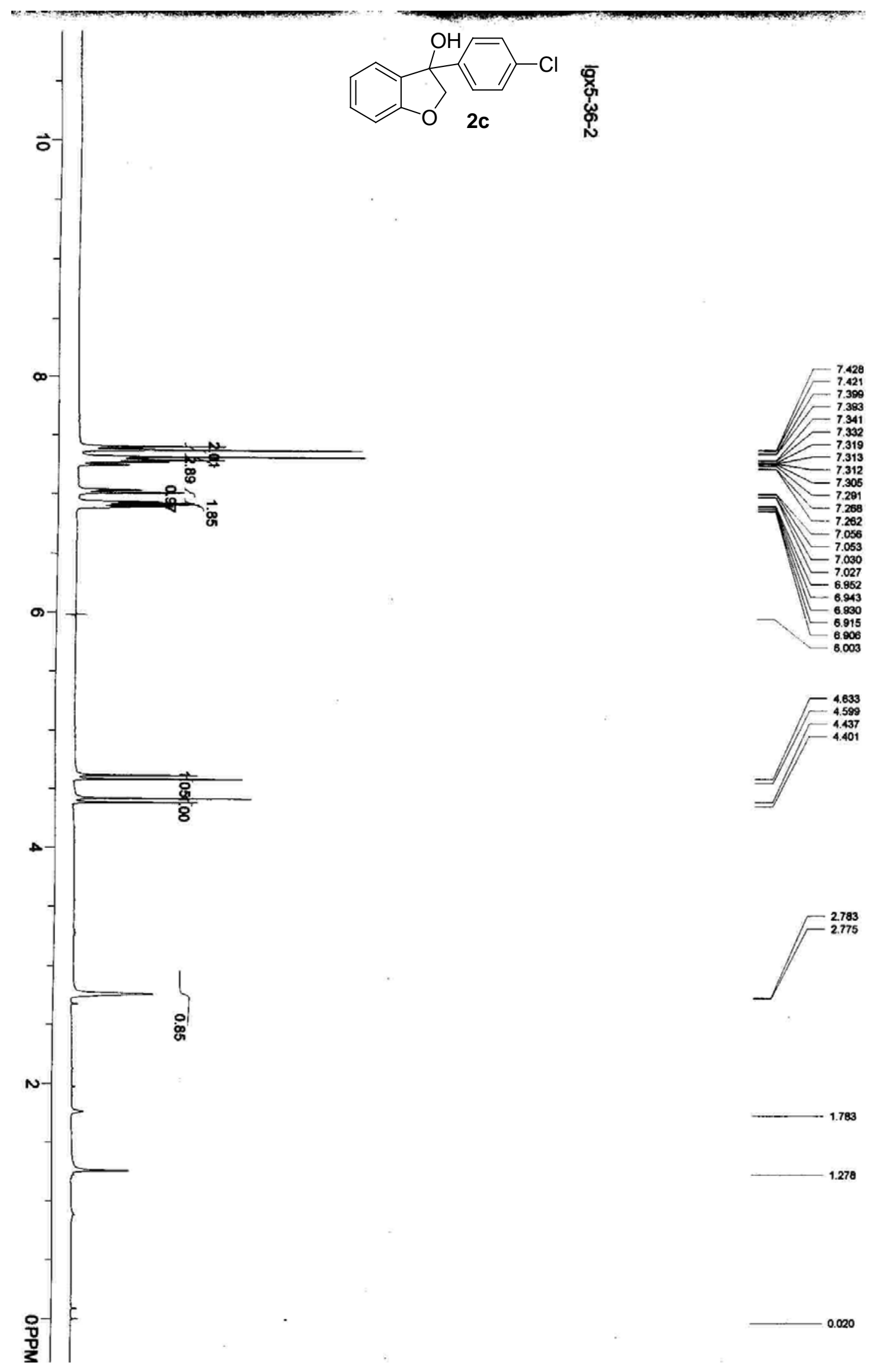



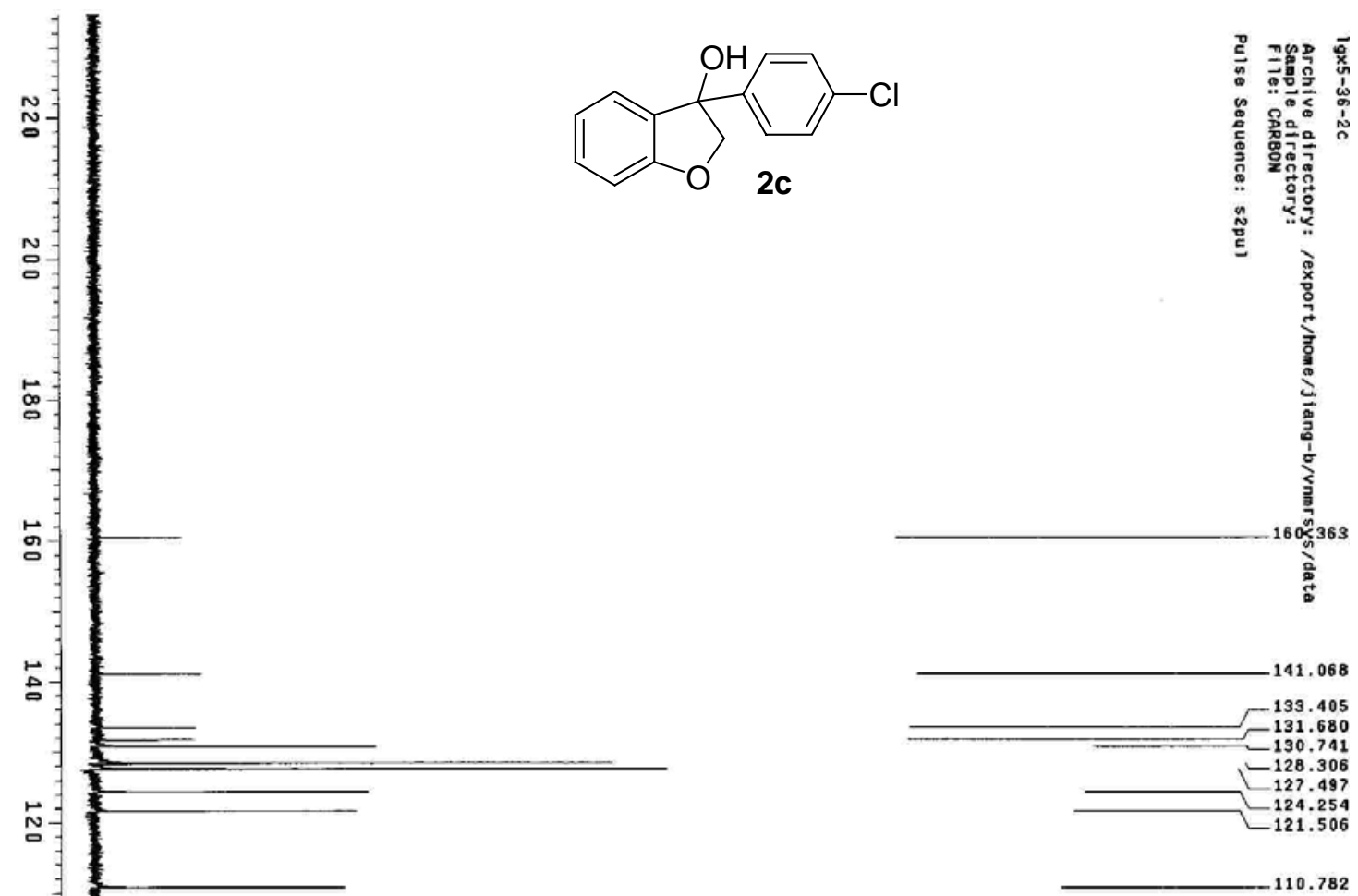

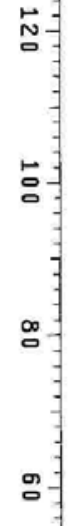

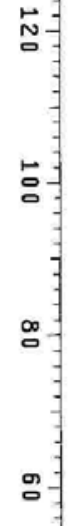

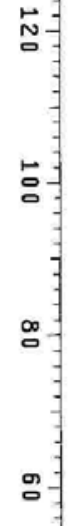

吕笺

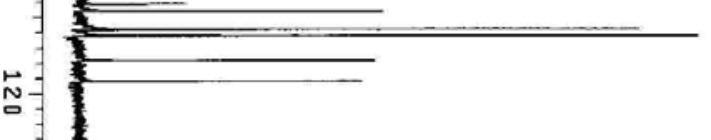

110.782
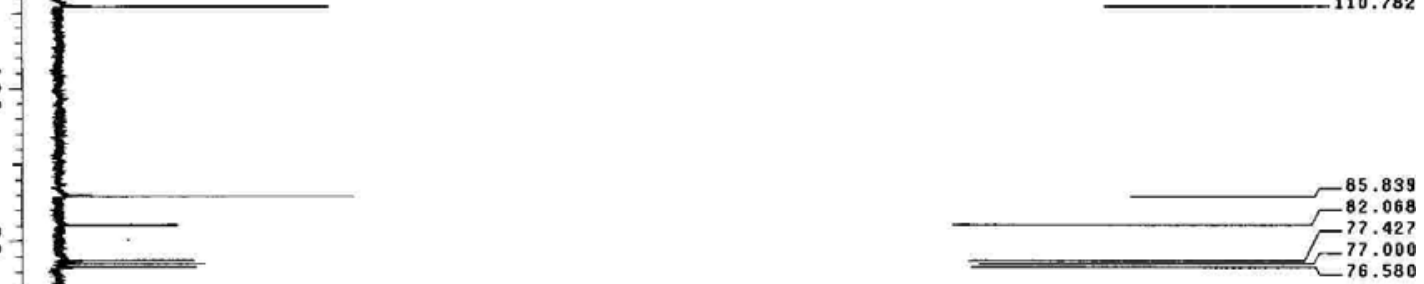

a $=$

ก-

穿等 

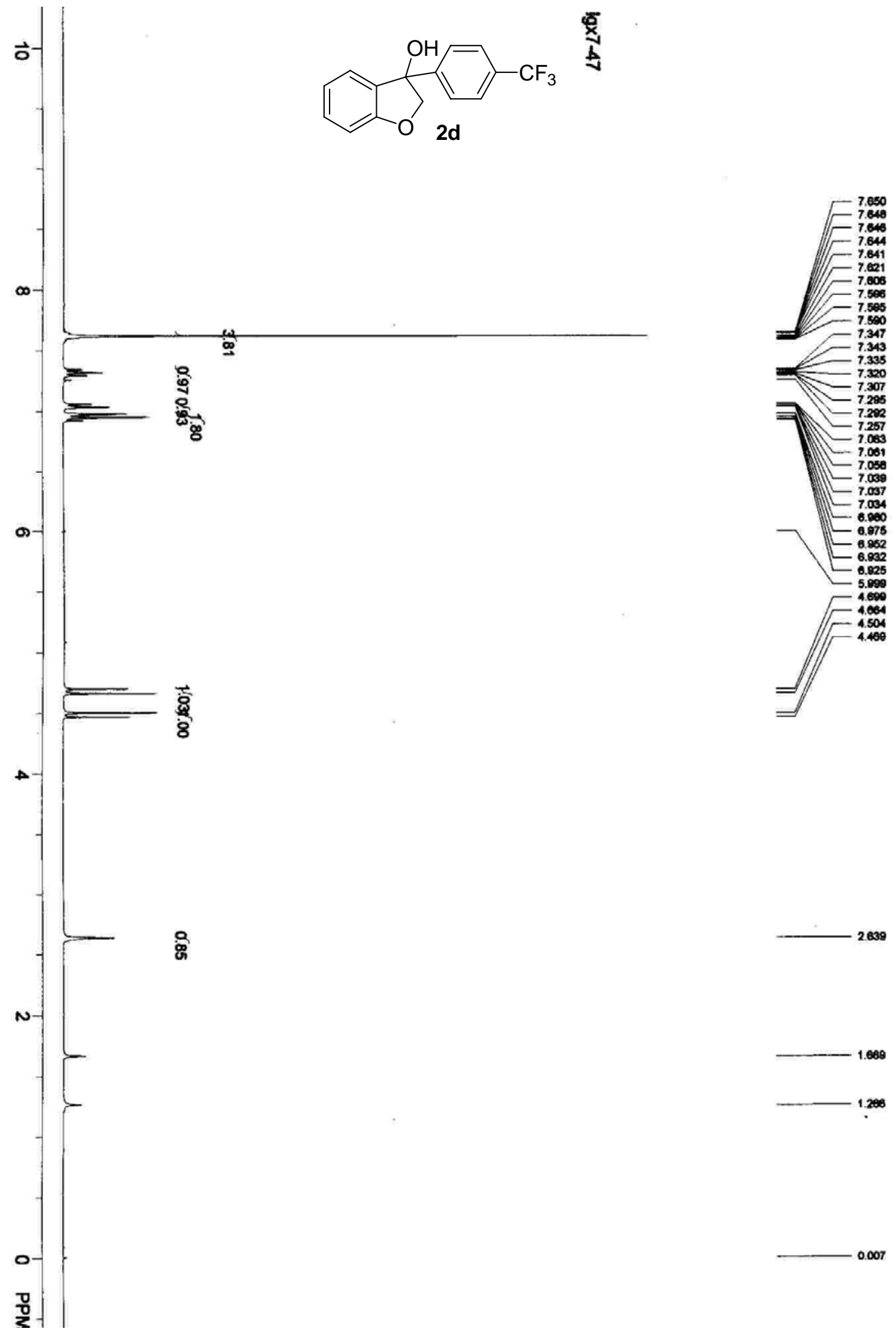

$-0.007$

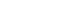




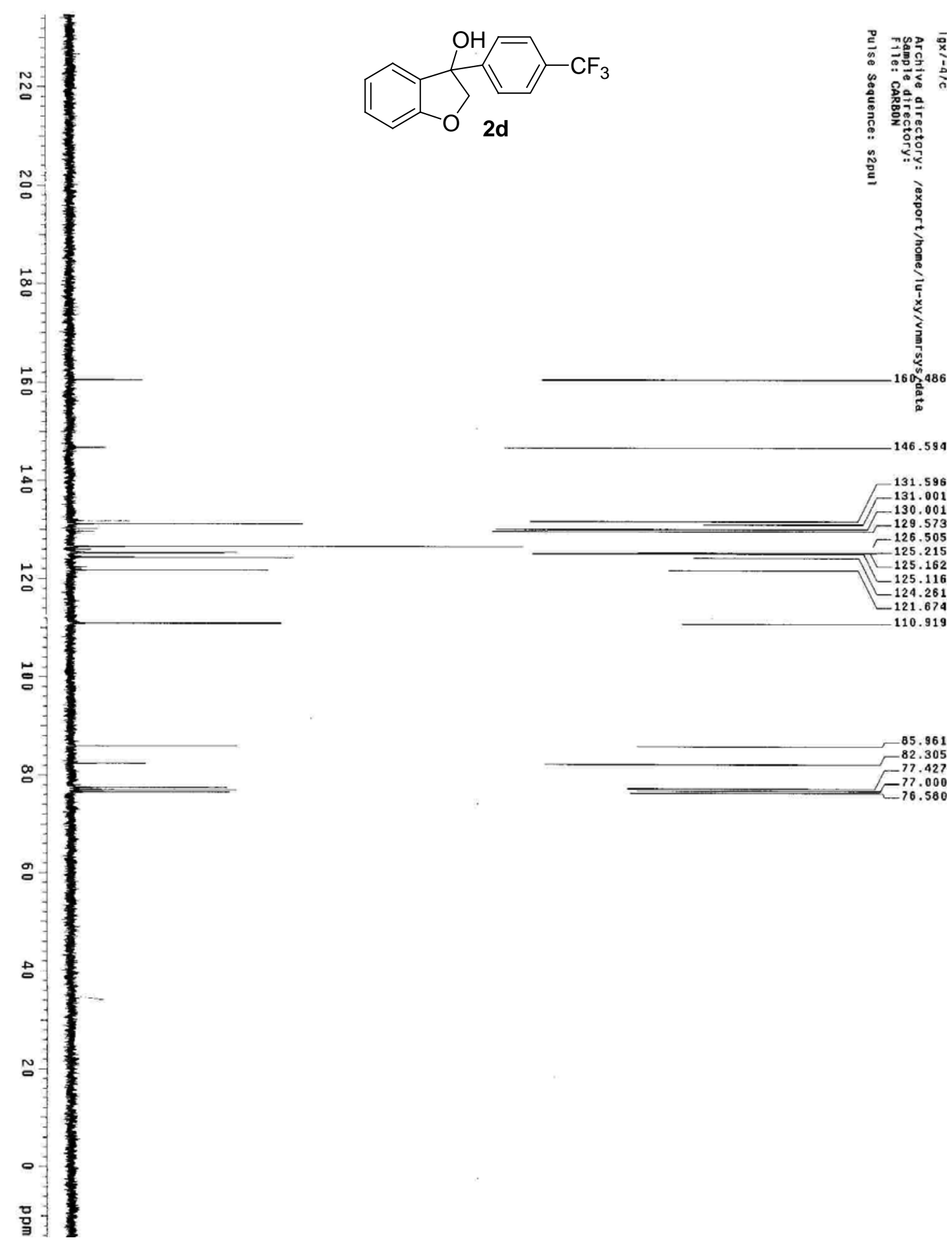




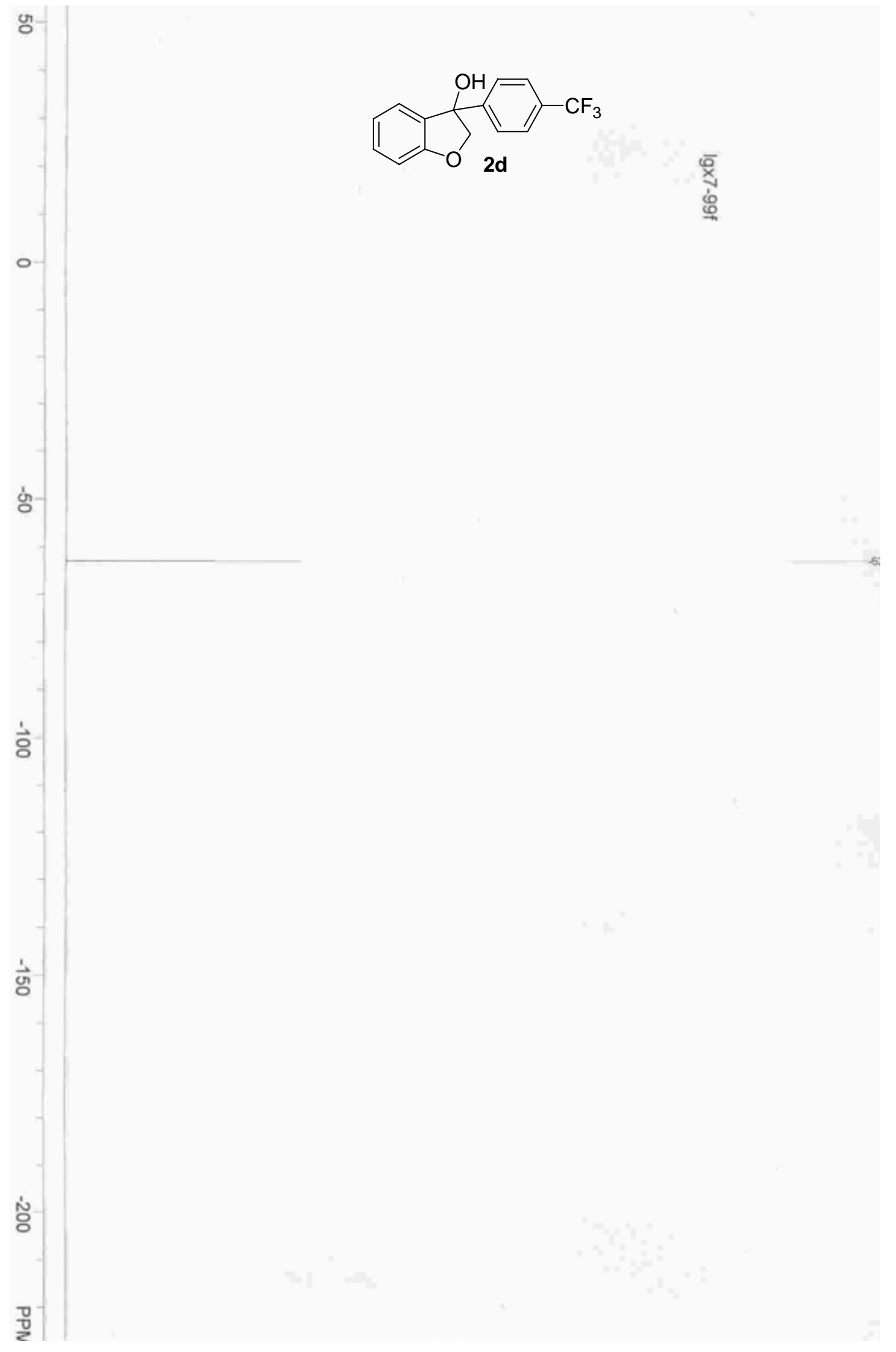




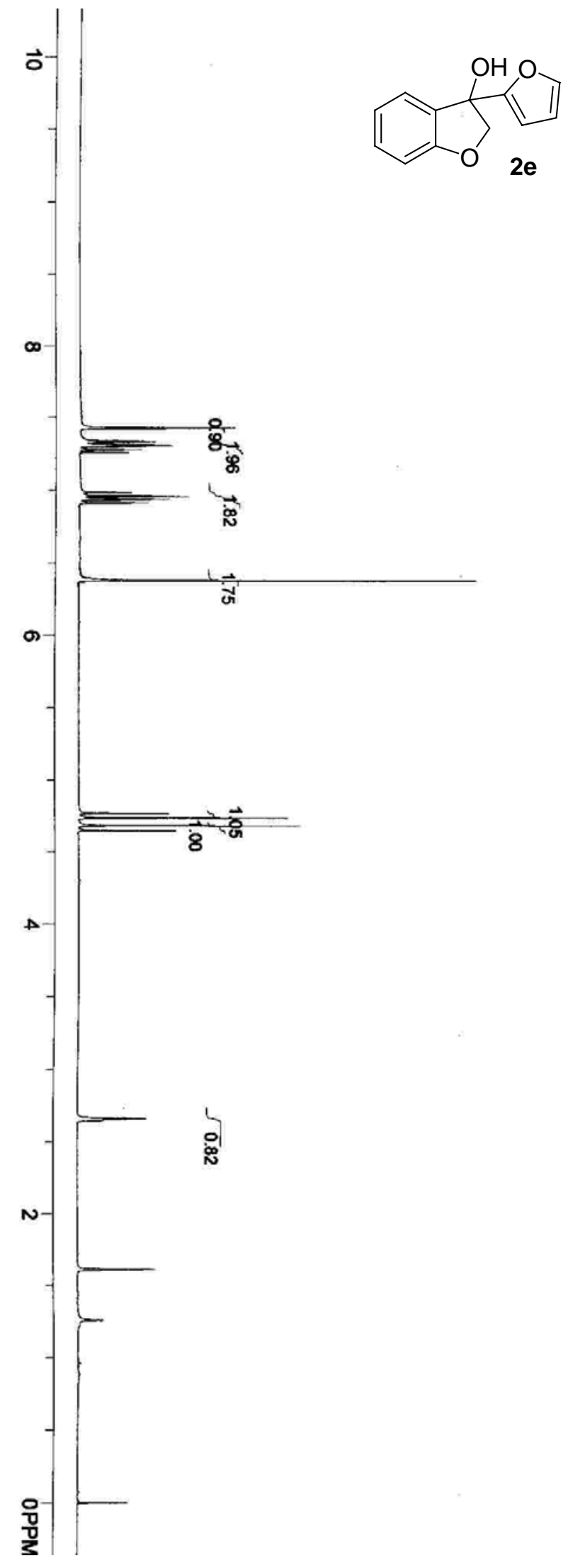

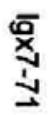
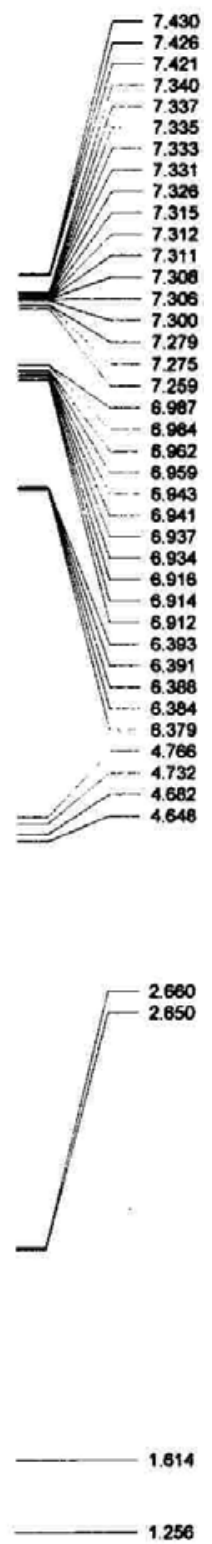


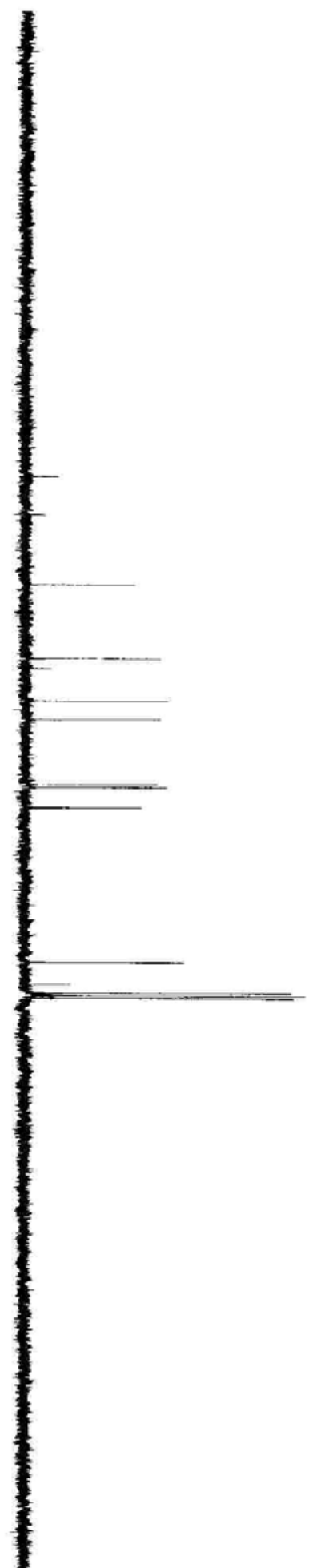

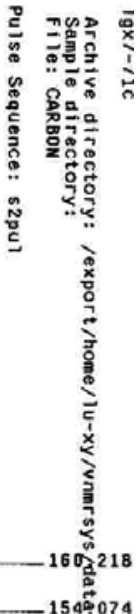

142,908
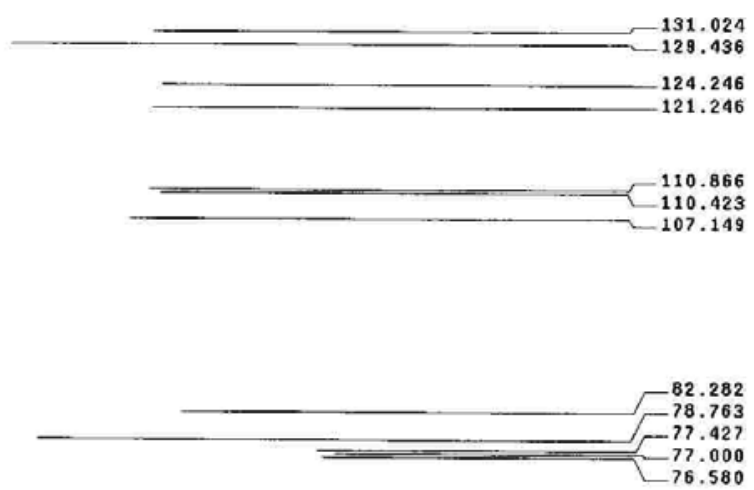


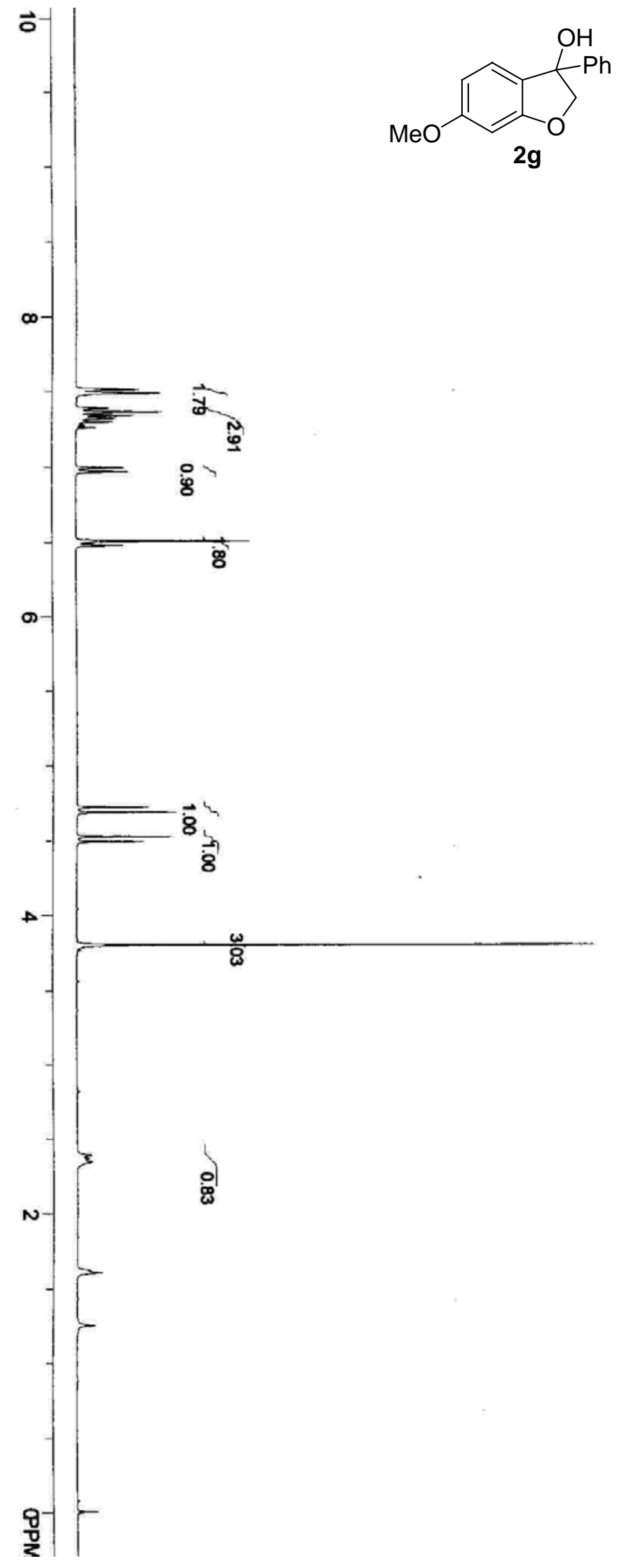

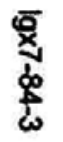
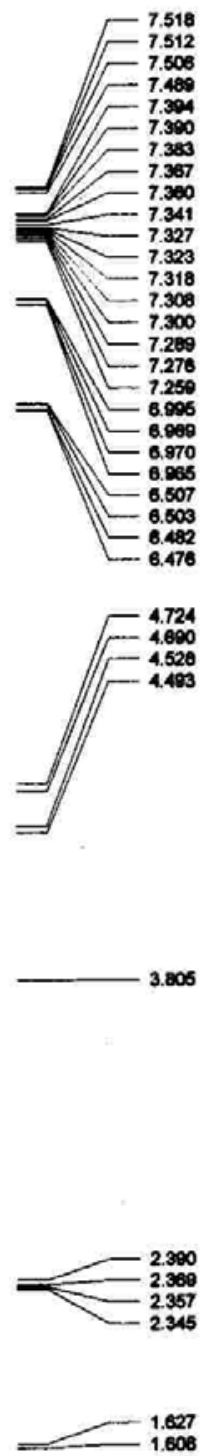

1.257

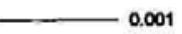




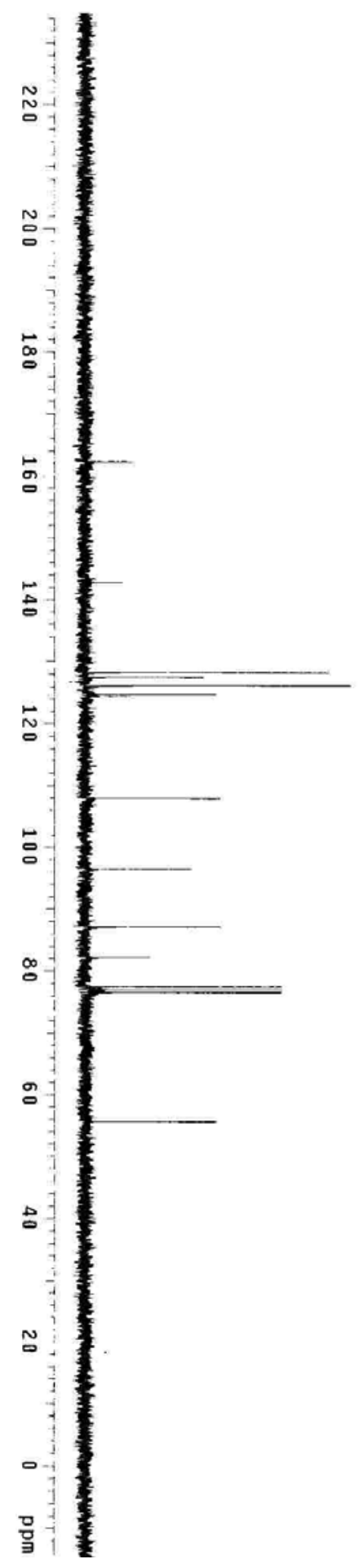

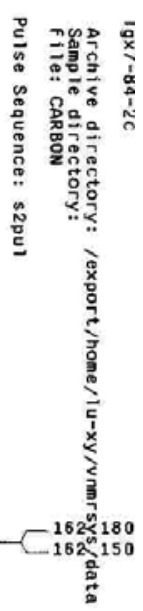

142.724

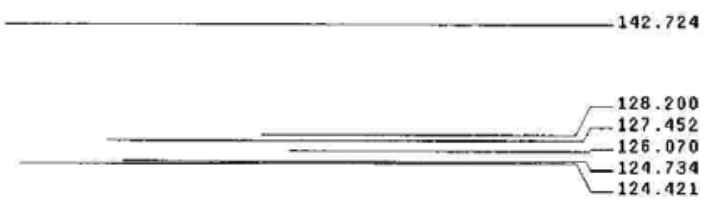

107.874

$-96.311$

87.052

$-82.190$ 71.420
-76.992

76.573
-76.992
-76

$-55.537$ 

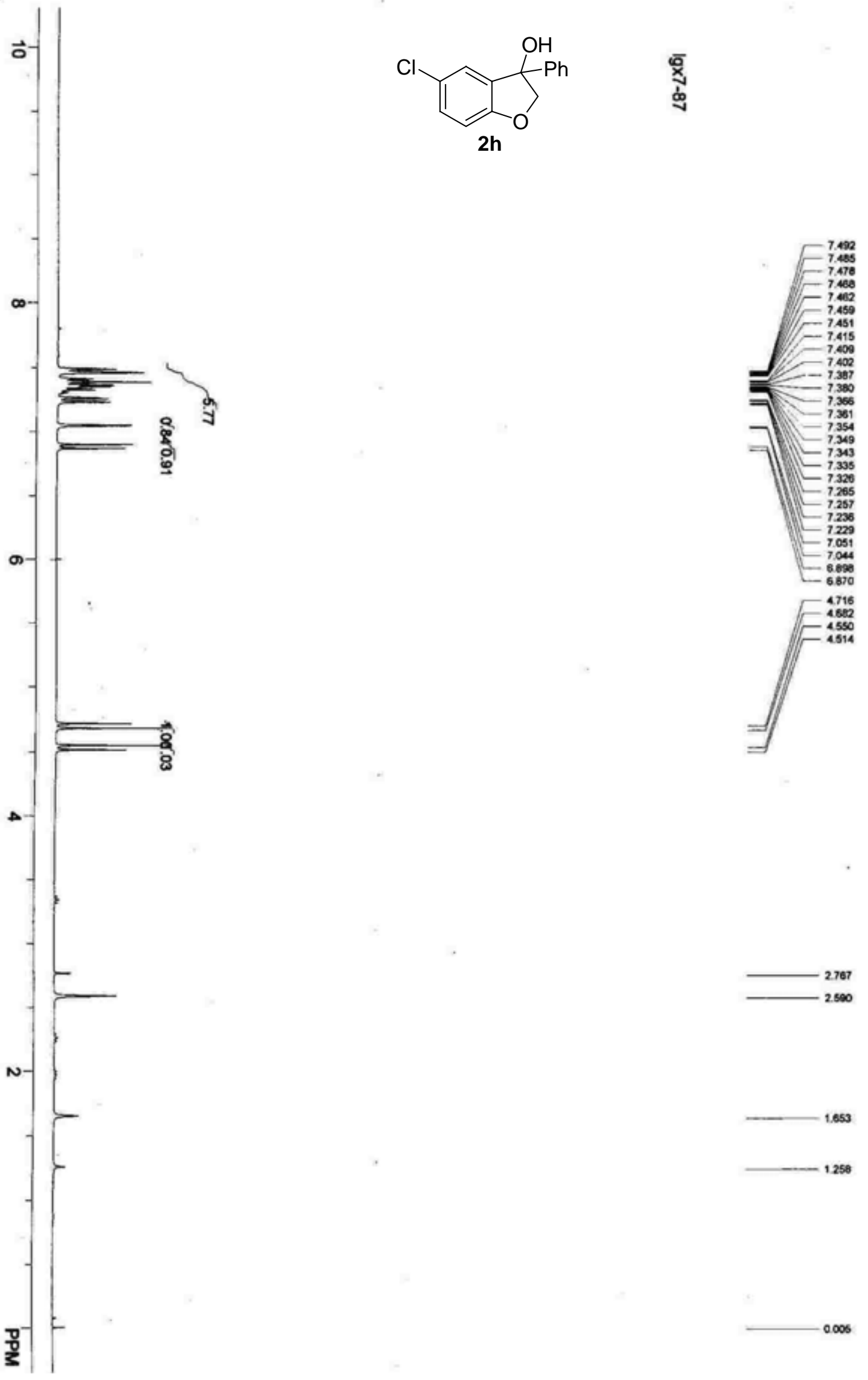


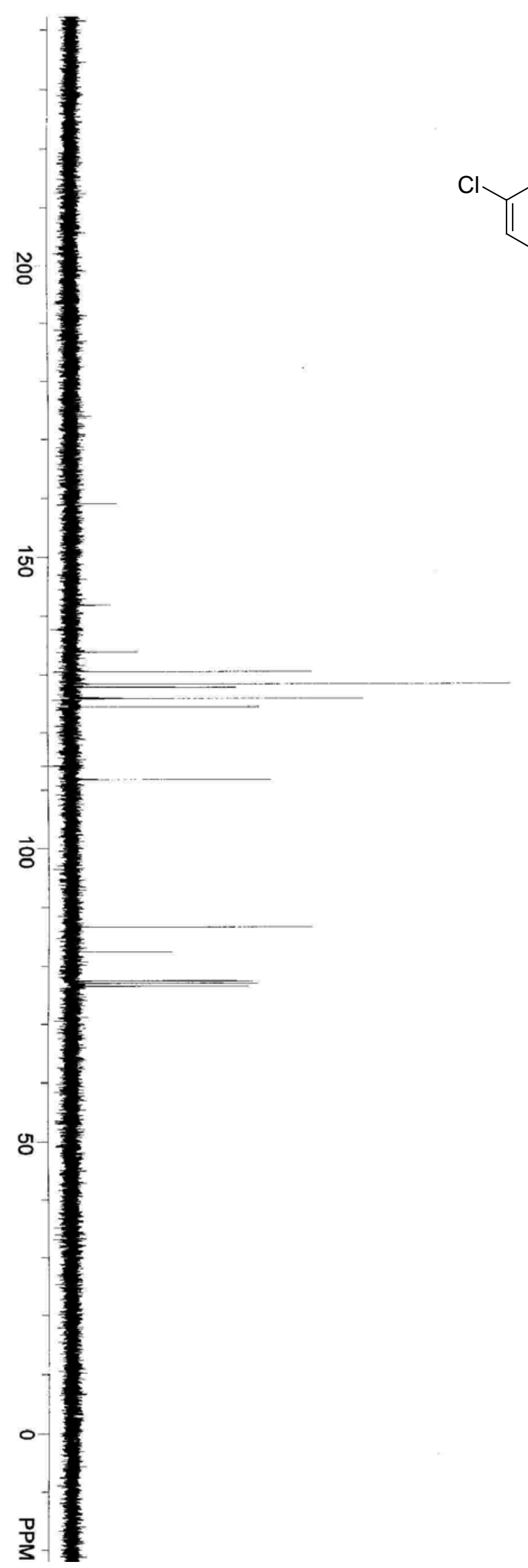

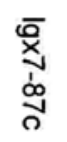

159090

141.808

133.900

- 130.528

128.384

127826

125.876

111873

.111 .873

-86.561
$-\quad 82.402$
77.408
76.988
76.946
76556

76556 

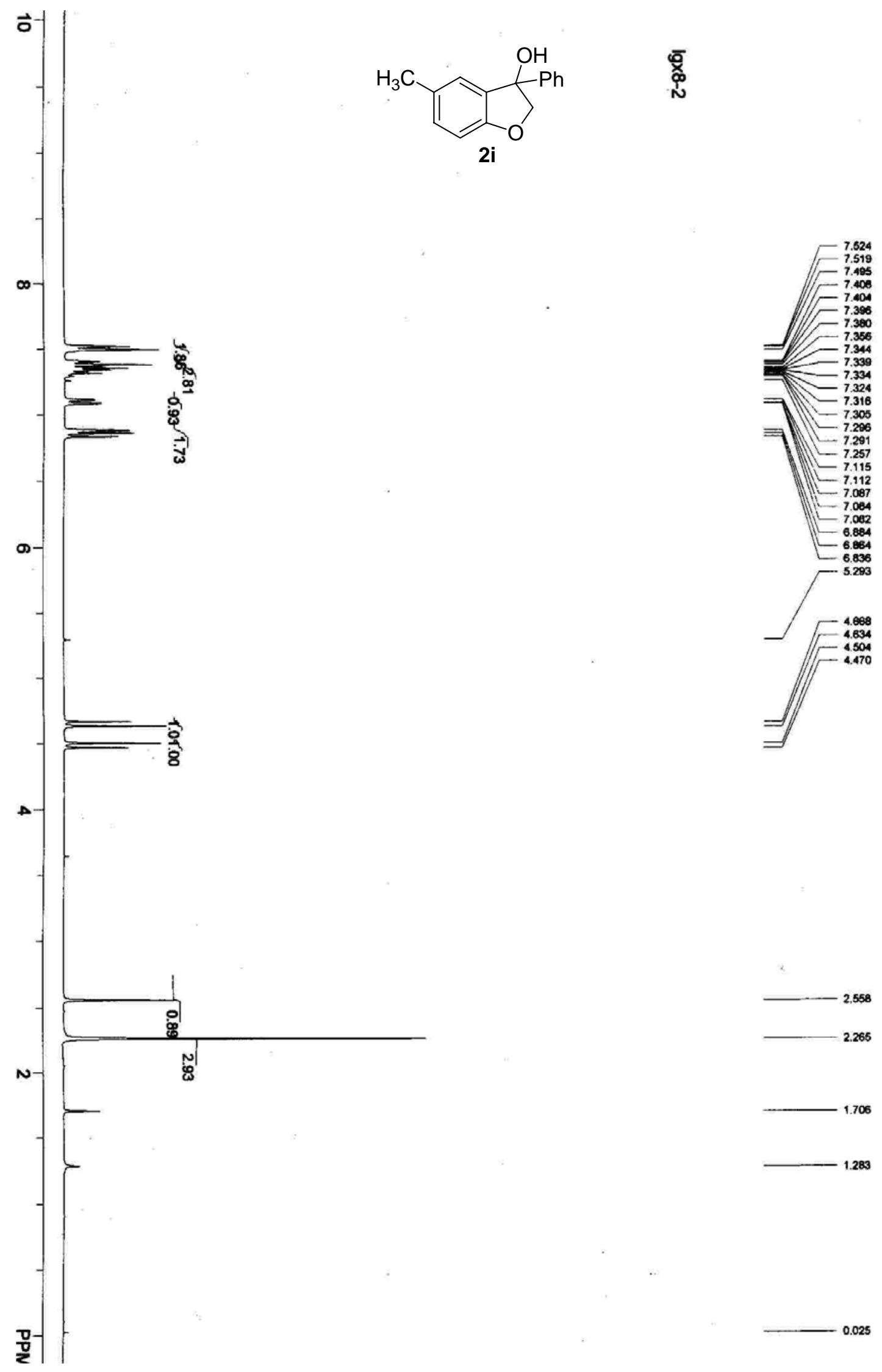

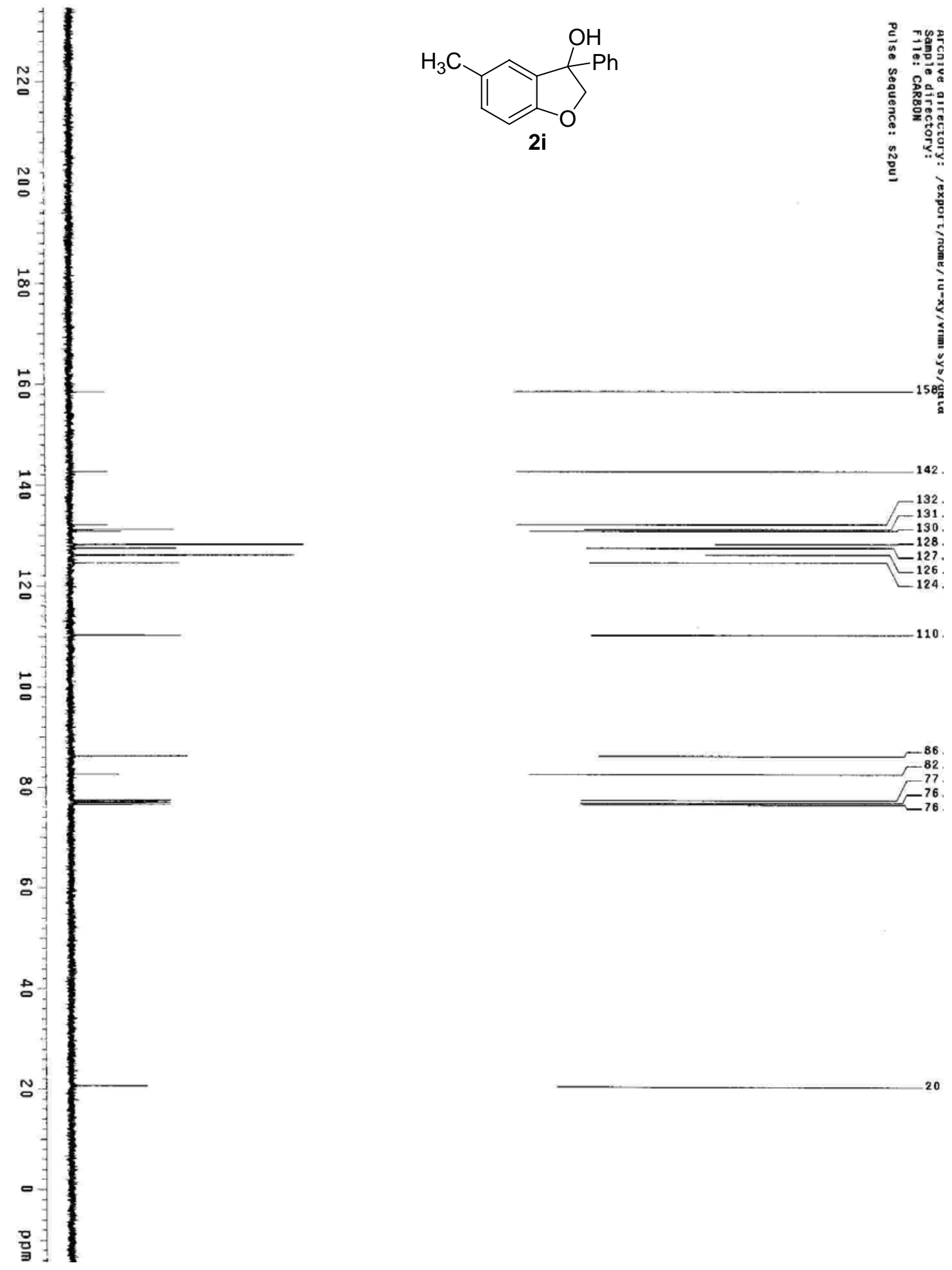

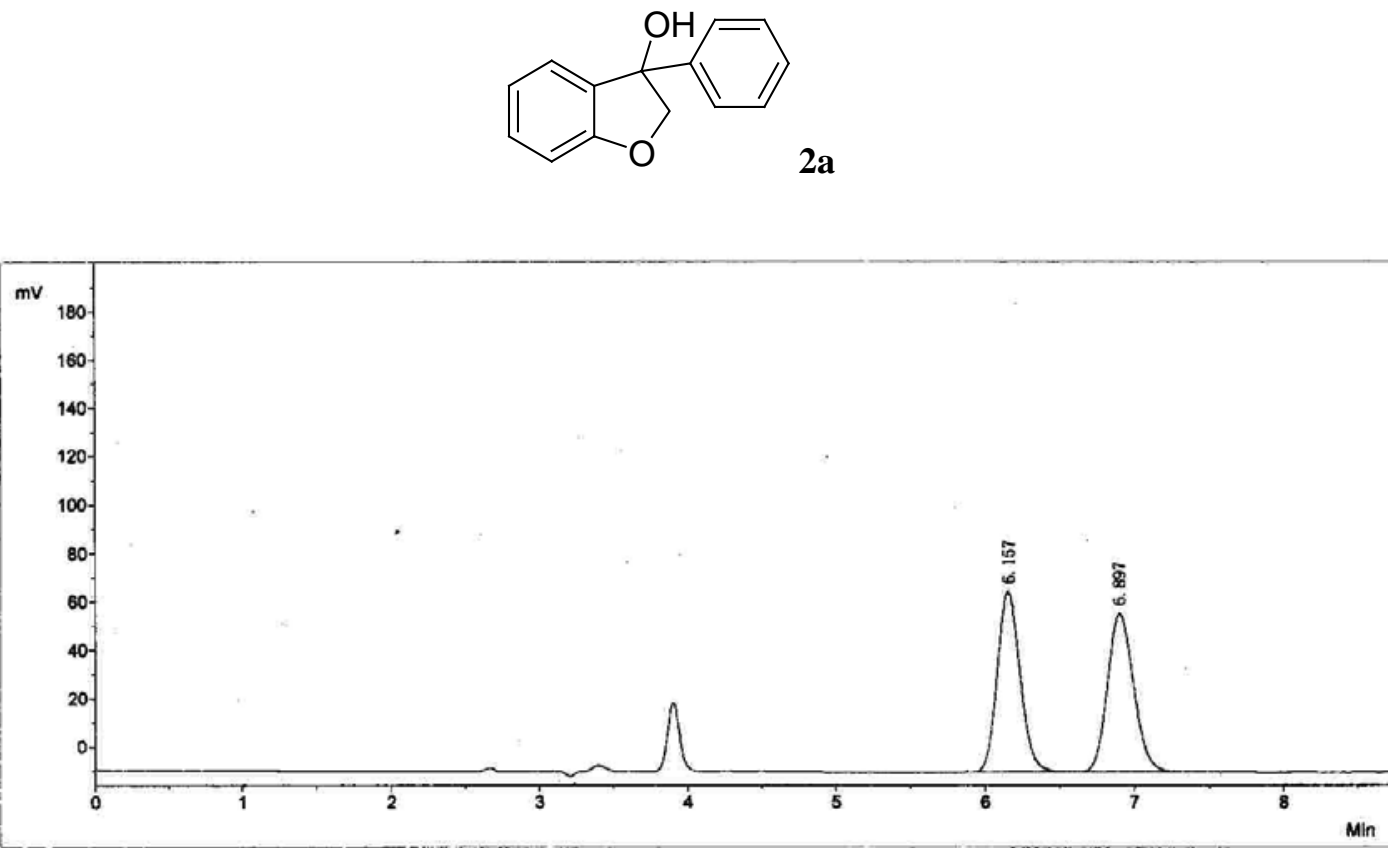

No. R. Time PeakHeight PeakArea PerCent

$\begin{array}{lllll}1 & 6.157 & 70023.4 & 796985.3 & 49.9425\end{array}$

$\begin{array}{lllll}2 & 6.897 & 62481.0 & 798821.2 & 50.0575\end{array}$

$\begin{array}{llll}\text { Total } & 132504.4 & 1595806.5 & 100.0000\end{array}$

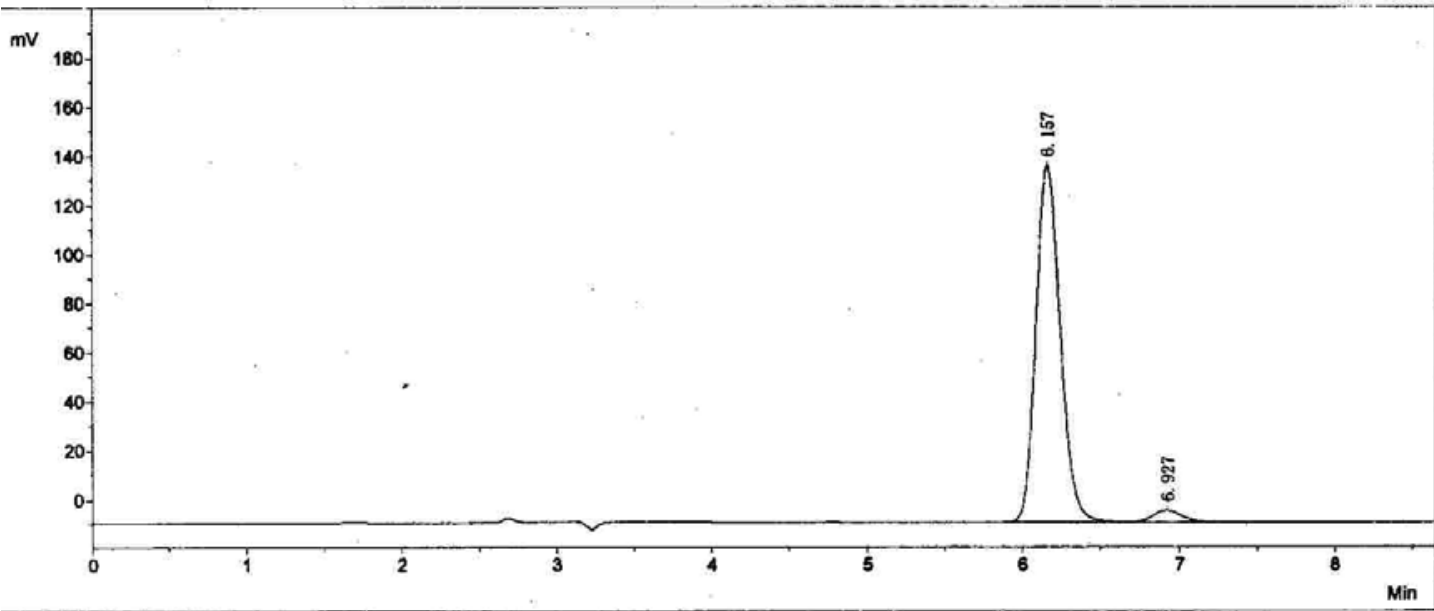

\begin{tabular}{rrrrr} 
No. & R. Time & PoakHeight & \multicolumn{1}{c}{ PoakArea } & \multicolumn{1}{c}{ PorCent } \\
\hline 1 & 6.157 & 140457.5 & 1574446.4 & 96.1164 \\
2 & 6.927 & 4945.7 & 63615.4 & 3.8836 \\
\hline Total & & 145403.2 & 1638061.8 & 100.0000
\end{tabular}



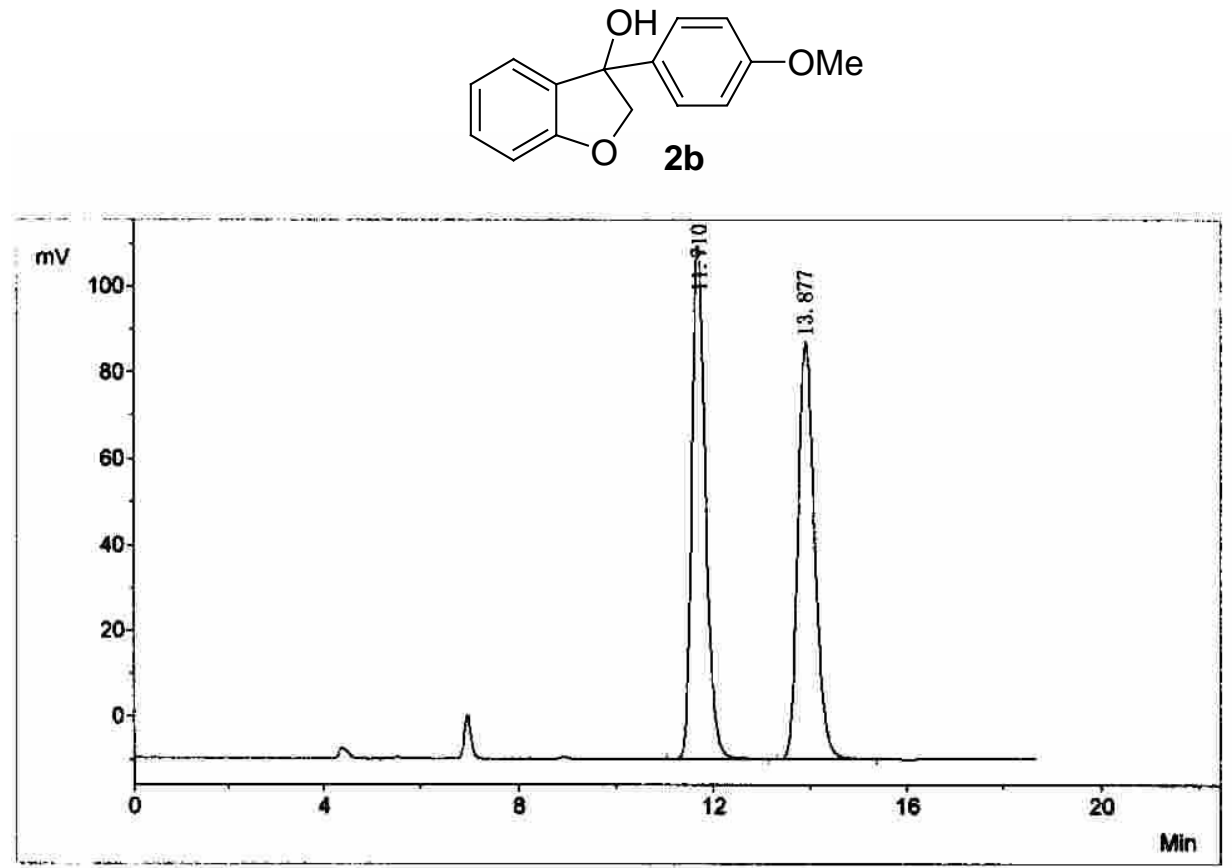

\begin{tabular}{rrrrrrr} 
No. PeakNo & ID. Name & R. Time & PeakHeight & PeakArea & PerCent \\
\hline 1 & 1 & Unknown & 11.710 & 115660.6 & 2348903.0 & 50.1267 \\
2 & 2 & Unknown & 13.877 & 95980.6 & 2337024.5 & 49.8733 \\
\hline Total & & & & 211641.2 & 4685927.5 & 100.0000
\end{tabular}

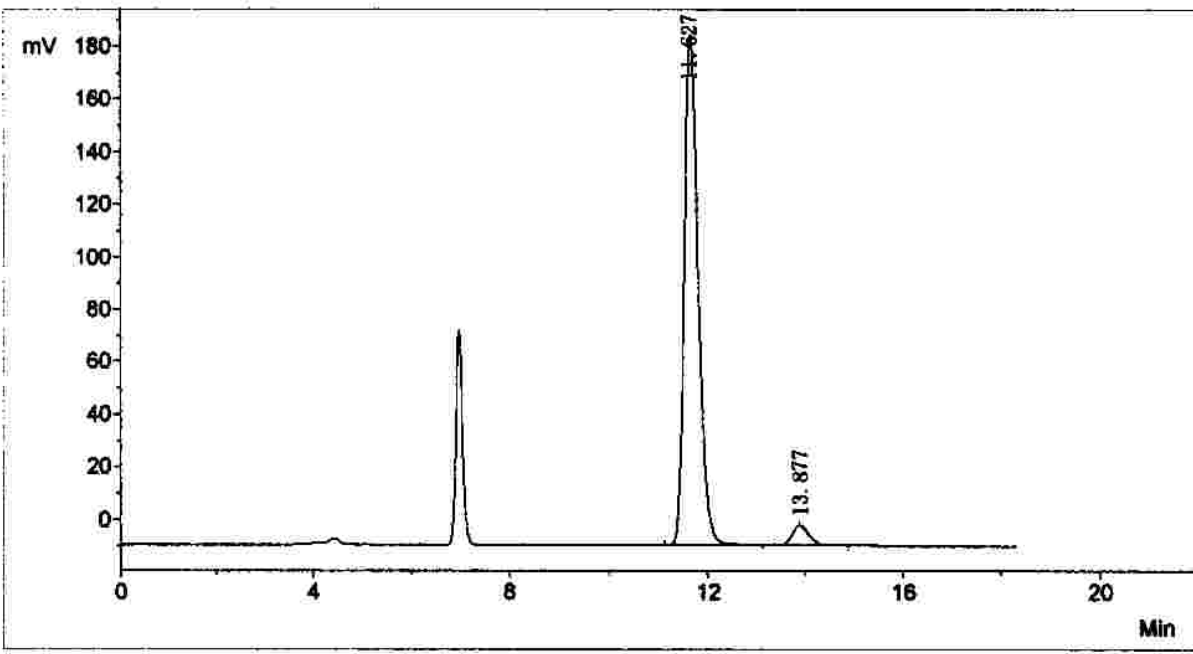

\begin{tabular}{rrrrrrr}
\multicolumn{1}{r}{ No. PeakNo } & ID. Name & R. Time & PeakHe ight & \multicolumn{1}{c}{ PeakArea } & \multicolumn{1}{c}{ PerCent } \\
\hline 1 & 1 & Unknown & 11.627 & 187867.5 & 3799607.5 & 95.5685 \\
2 & 2 & Unknown & 13.877 & 7395.3 & 176186.3 & 4.4315 \\
\hline
\end{tabular}


<smiles>Oc1ccccc1C1(O)COc2ccccc21</smiles>

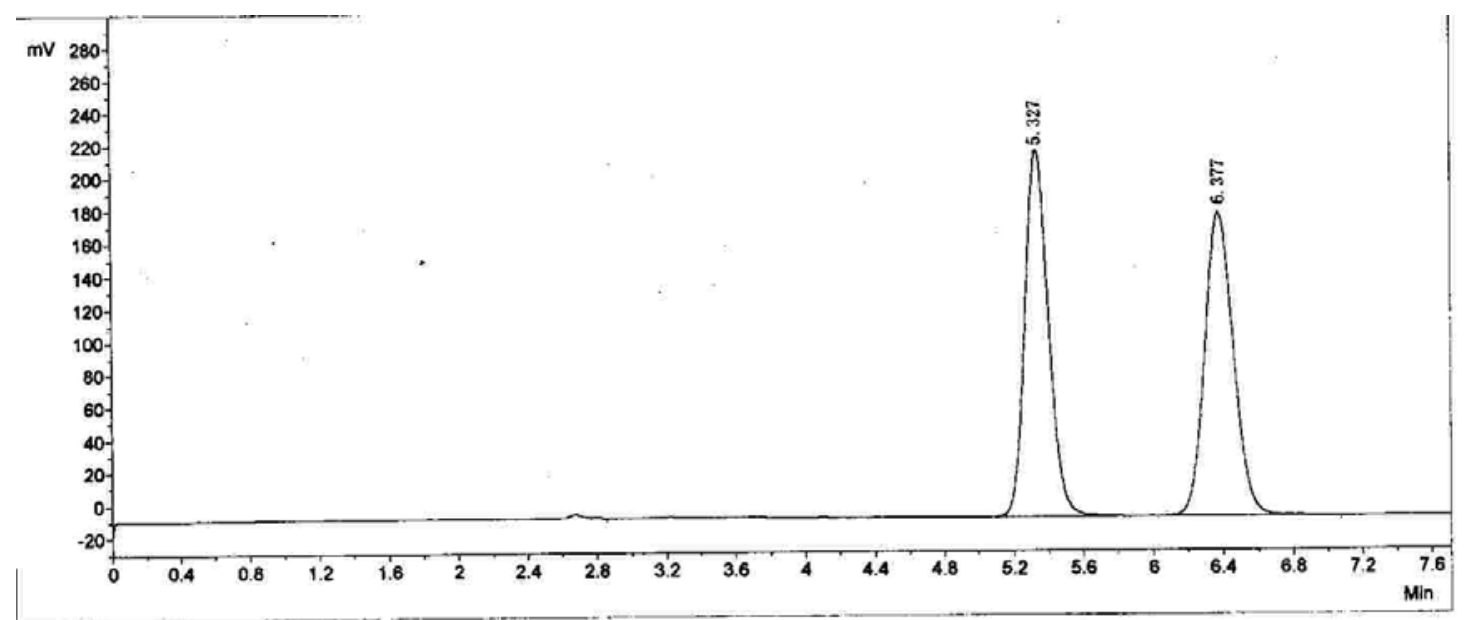

\begin{tabular}{rrrrr}
\multicolumn{1}{r}{ No. } & R. Time & PeakHeight & PeakArea & \multicolumn{1}{c}{ PerCent } \\
\hline 1 & 5.327 & 221298.0 & 2104060.6 & 50.0643 \\
2 & 6.377 & 184476.9 & 2098654.2 & 49.9357 \\
\hline Total & & 405774.9 & 4202714.8 & 100.0000
\end{tabular}

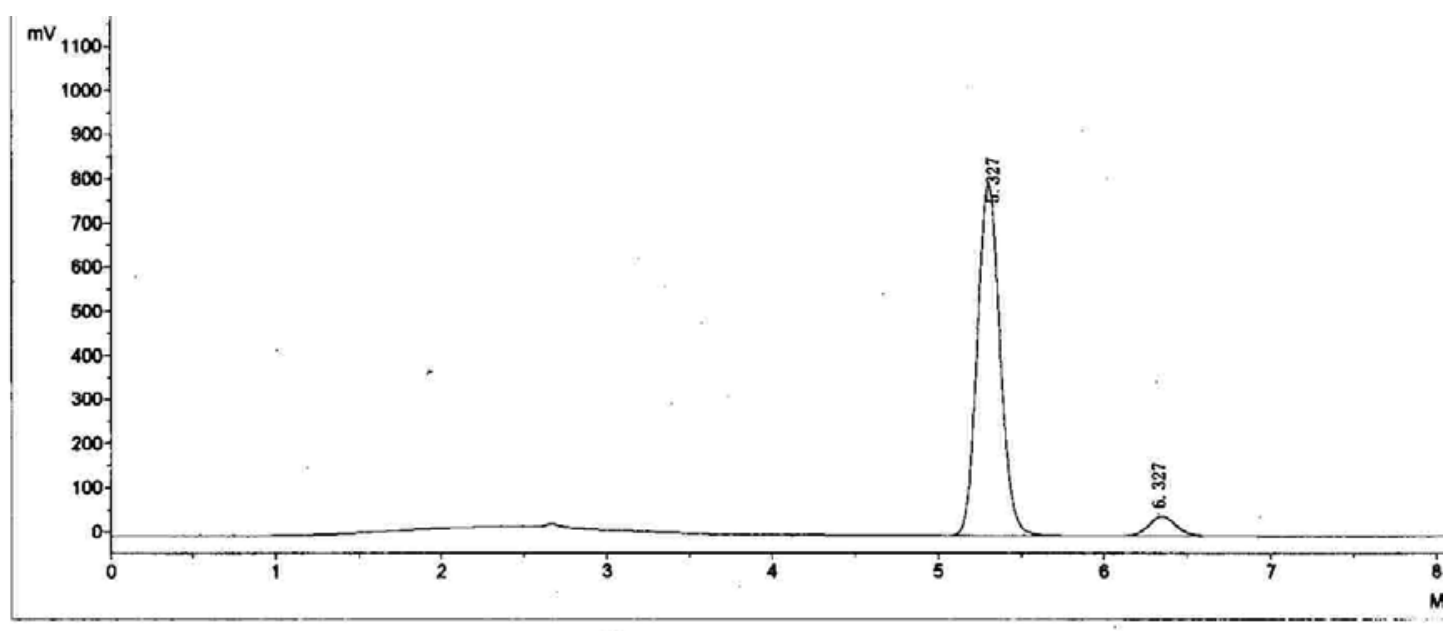

\begin{tabular}{rrrrr} 
No. & R. Time & PeakHeight & \multicolumn{1}{c}{ PeakArea } & \multicolumn{1}{c}{ PerCont } \\
\hline 1 & 5.327 & 734431.6 & 7624087.2 & 93.7164 \\
2 & 6.327 & 42621.6 & 511191.3 & 6.2836 \\
\hline Total & & 777053.2 & 8135278.5 & 100.0000
\end{tabular}



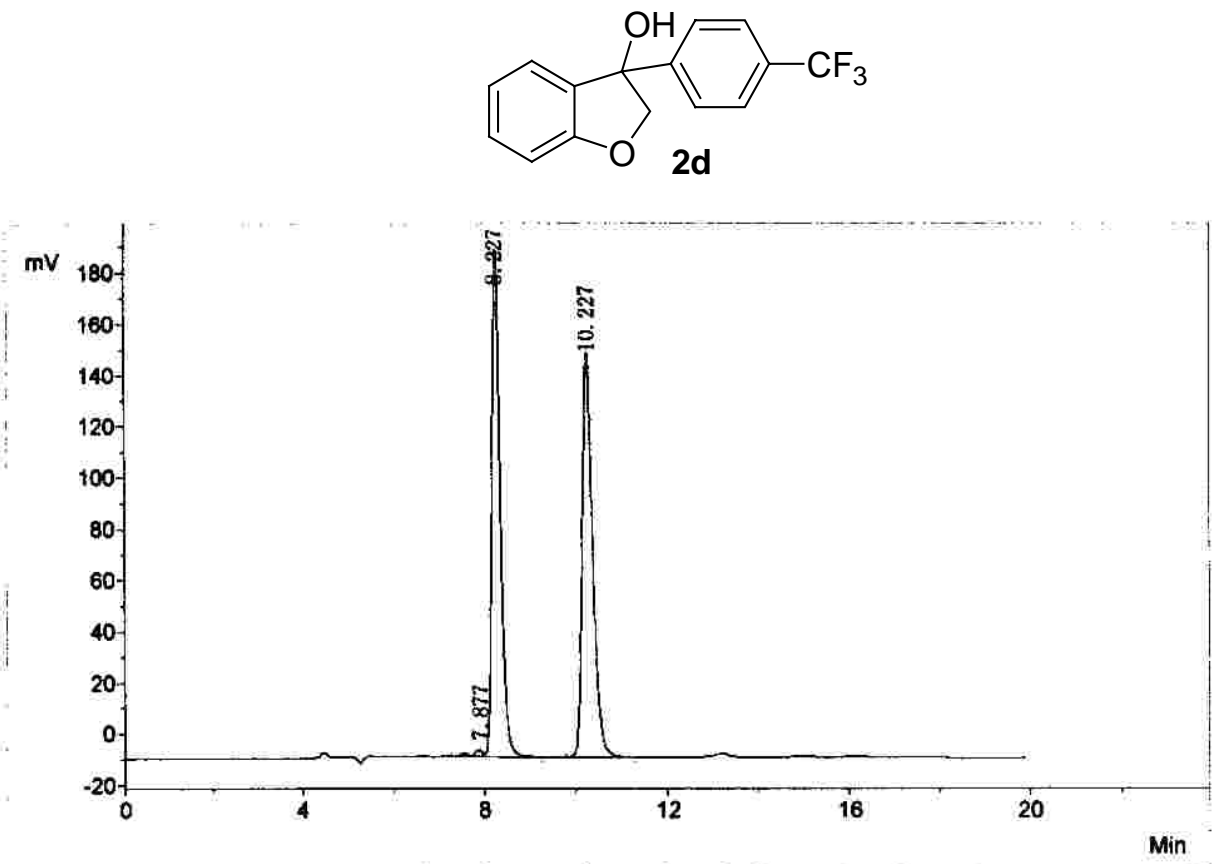

$\begin{array}{rrrrrrr}\text { No. PeakNo } & \text { ID. Name } & \text { R. Time } & \text { PeakHe ight } & \text { PeakArea } & \text { PerCent } \\ 1 & 1 & \text { Unknown } & 7.877 & 2451.3 & 39765.1 & 0.7863 \\ 2 & 2 & \text { Unknown } & 8.227 & 188347.8 & 2490402.7 & 49.2447 \\ 3 & 3 & \text { Unknown } & 10.227 & 153855.8 & 2527036.5 & 49.9690\end{array}$

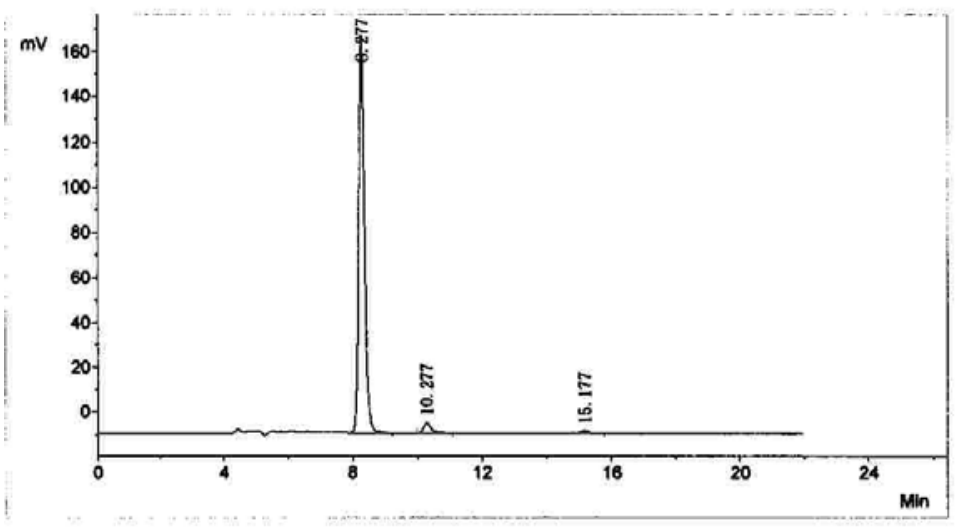

\begin{tabular}{rrrrrrr}
\multicolumn{2}{r}{ No. PeakNo } & ID. Name & R. Time & PeakHeight & \multicolumn{1}{c}{ PeakArea } & \multicolumn{1}{c}{ PerCent } \\
\hline 1 & 1 & Unknown & 8.277 & 171570.2 & 2204547.3 & 96.0630 \\
2 & 2 & Unknown & 10.277 & 4392.1 & 74346.5 & 3.2396 \\
3 & 3 & Unknown & 15.177 & 580.4 & 16003.1 & 0.6973 \\
Tnt.al & & & & 176542.7 & 22.94896 .9 & $100.00 n 0$
\end{tabular}




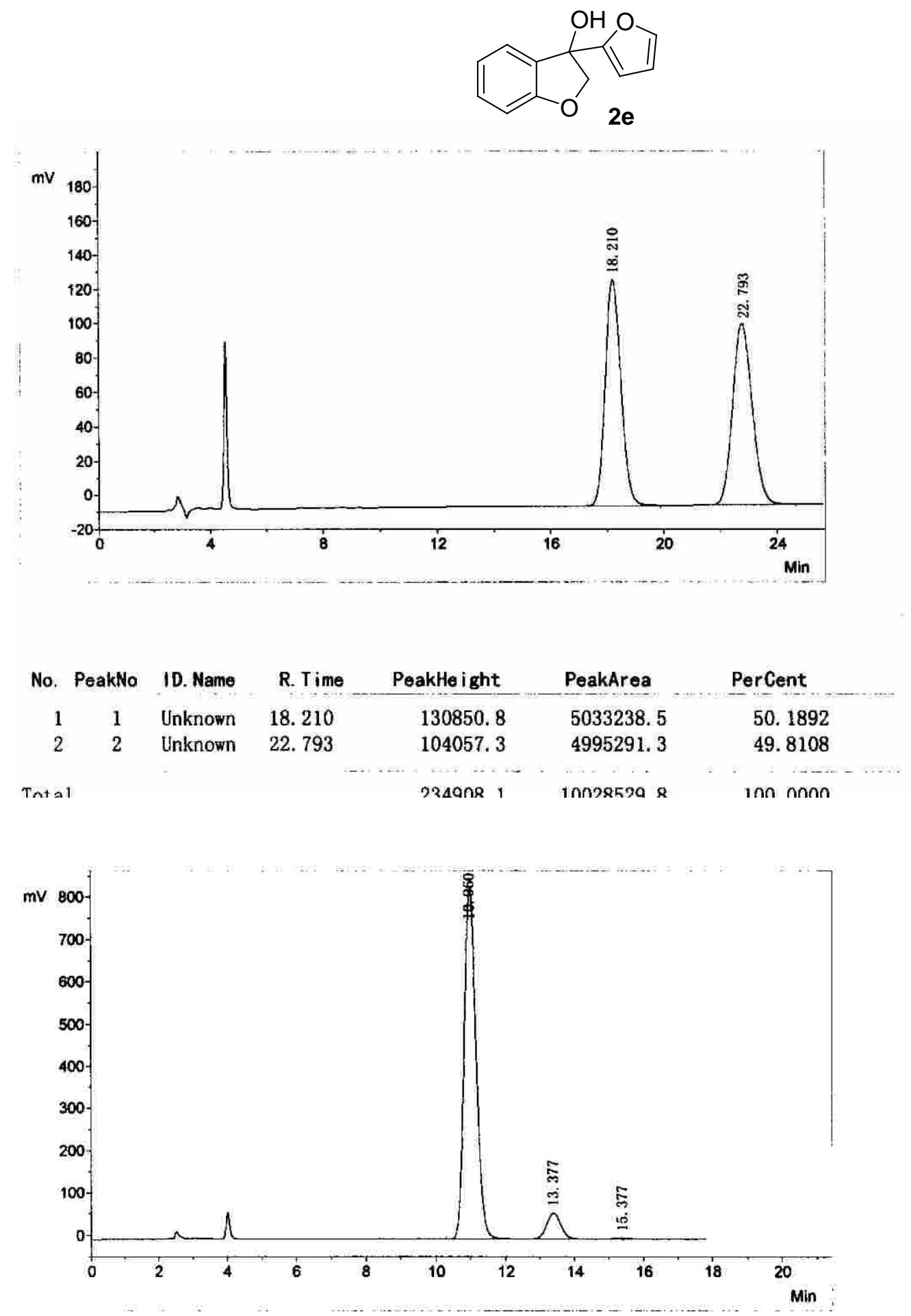

\begin{tabular}{|c|c|c|c|c|c|c|}
\hline No. & PeakNo & ID. Name & R. T ime & PeakHeight & PeakArea & PerCent \\
\hline 1 & 1 & Unknown & 10.960 & 813972.3 & 19679698. 1 & 91.7940 \\
\hline 2 & 2 & Unknown & 13. 377 & 60208.8 & 1712867.1 & 7.9895 \\
\hline 3 & 3 & Unknown & 15. 377 & 1394.6 & 46421. 4 & 0.2165 \\
\hline Total & & & & 875575.6 & 21438986.6 & 100.0000 \\
\hline
\end{tabular}



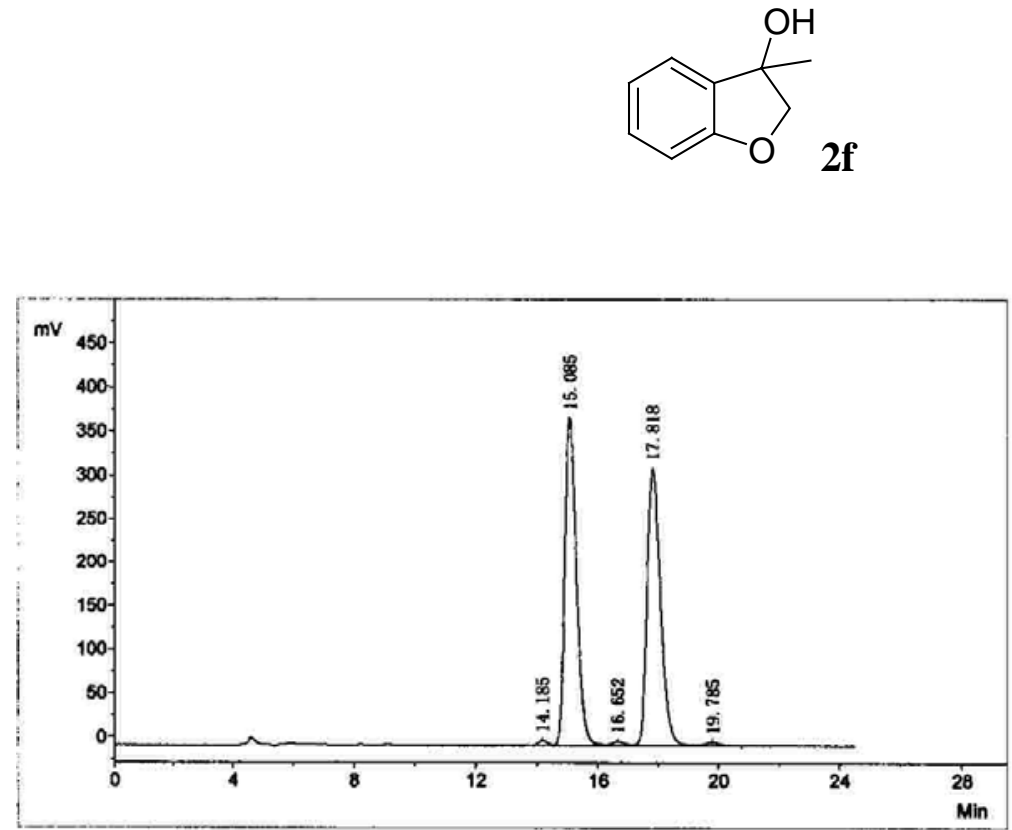

\begin{tabular}{rrrrrrr}
\multicolumn{2}{r}{ No. PeakNo } & ID. Name & R. Time & PeakHeight & \multicolumn{1}{c}{ PeakArea } & PerCent \\
\hline 1 & 1 & Unknown & 14.185 & 5152.1 & 118184.1 & 0.5853 \\
2 & 2 & Unknown & 15.085 & 374543.4 & 9976158.3 & 49.4051 \\
3 & 3 & Unknown & 16.652 & 4750.8 & 176940.7 & 0.8763 \\
4 & 4 & Unknown & 17.818 & 316422.0 & 9787023.3 & 48.4685 \\
5 & 5 & Unknown & 19.785 & 3947.9 & 134252.2 & 0.6649 \\
\hline Total & & & & 704816.1 & 20192558.6 & 100.0000
\end{tabular}

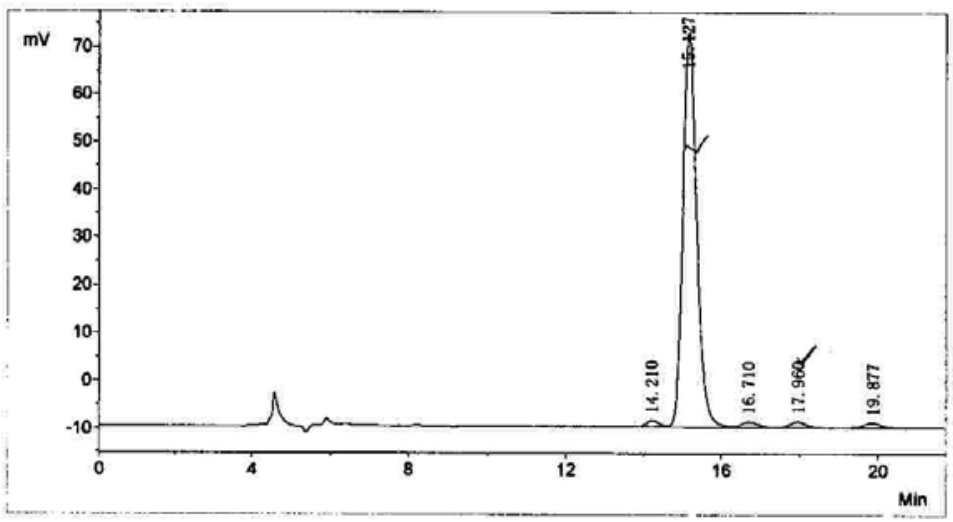

\begin{tabular}{rrrlrrr} 
No. PeakNo & ID. Name & R. Time & PeakHeight & \multicolumn{1}{c}{ PeakArea } & \multicolumn{1}{c}{ PerCent } \\
\hline 1 & 1 & Unknown & 14.210 & 1261.0 & 28414.8 & 1.3067 \\
2 & 2 & Unknown & 15.127 & 79911.6 & 2047387.0 & 94.1494 \\
3 & 3 & Unknown & 16.710 & 1131.3 & 34963.4 & 1.6078 \\
4 & 4 & Unknown & 17.960 & 1179.3 & 33805.3 & 1.5545 \\
5 & 5 & Unknown & 19.877 & 968.5 & 30045.1 & 1.3816 \\
\hline Total & & & 84451.8 & 2174615.6 & 100.0000
\end{tabular}


<smiles>COc1ccc2c(c1)OCC2(O)c1ccccc1</smiles>

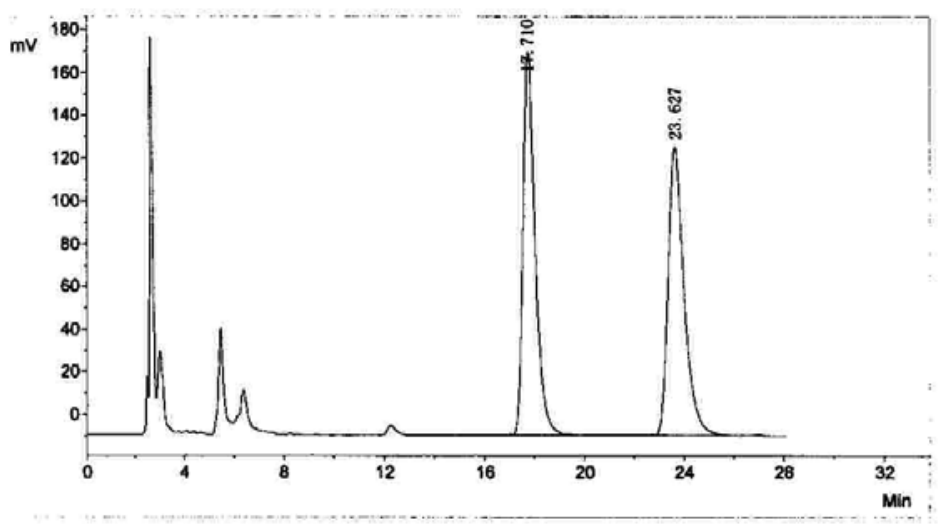

\begin{tabular}{|c|c|c|c|c|c|c|}
\hline No. & PeakNo & 10. Name & R. Time & PeakHeight & PeakArea & PerCent \\
\hline 1 & 1 & Unknown & 17. 710 & 176318.8 & 6018750.0 & 49. 9552 \\
\hline 2 & 2 & Unknown & 23.627 & 134870.2 & 6029545.9 & 50.0448 \\
\hline
\end{tabular}

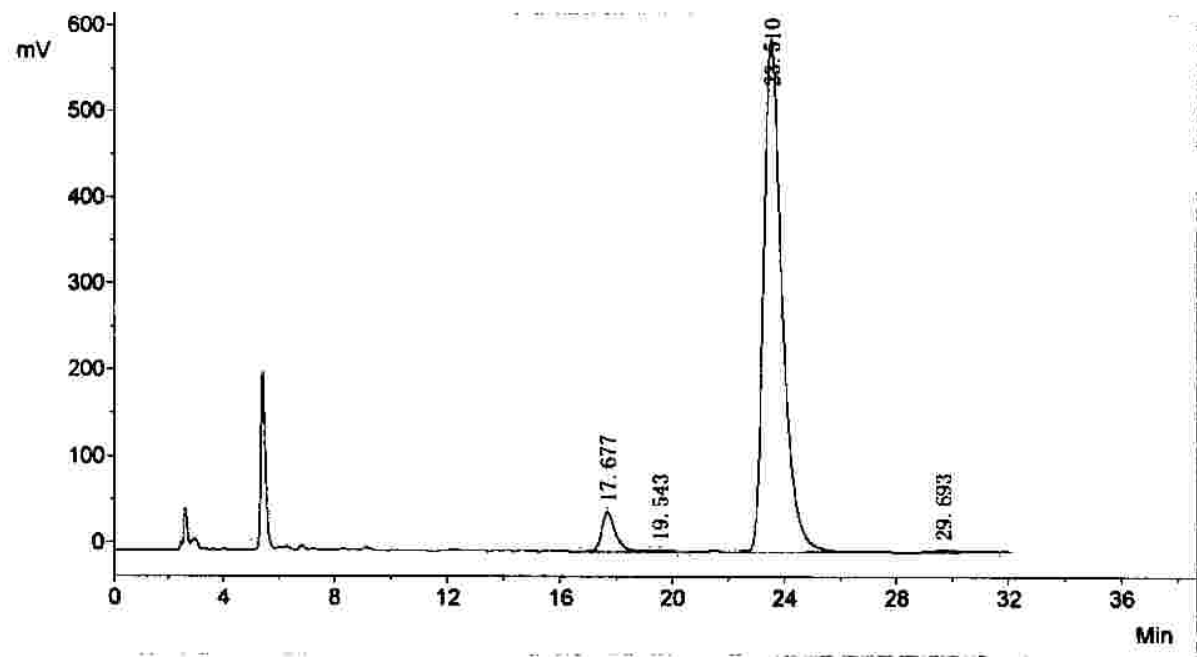

\begin{tabular}{ccccrrr}
\multicolumn{2}{c}{ No. PeakNo } & ID. Name & R. Time & PeakHeight & PeakArea & \multicolumn{1}{c}{ PerCent } \\
1 & 1 & Unknown & 17.677 & 44841.6 & 1509404.2 & 5.2250 \\
2 & 2 & Unknown & 19.543 & 301.4 & 9563.1 & 0.0331 \\
3 & 3 & Unknown & 23.510 & 592918.6 & 27262637.1 & 94.3724 \\
4 & 4 & Unknown & 29.693 & 1663.0 & 106741.8 & 0.3695 \\
\hline & & & & 639724.5 & 28888346.2 & 100.0000
\end{tabular}


<smiles>OC1(c2ccccc2)COc2ccc(Cl)cc21</smiles>

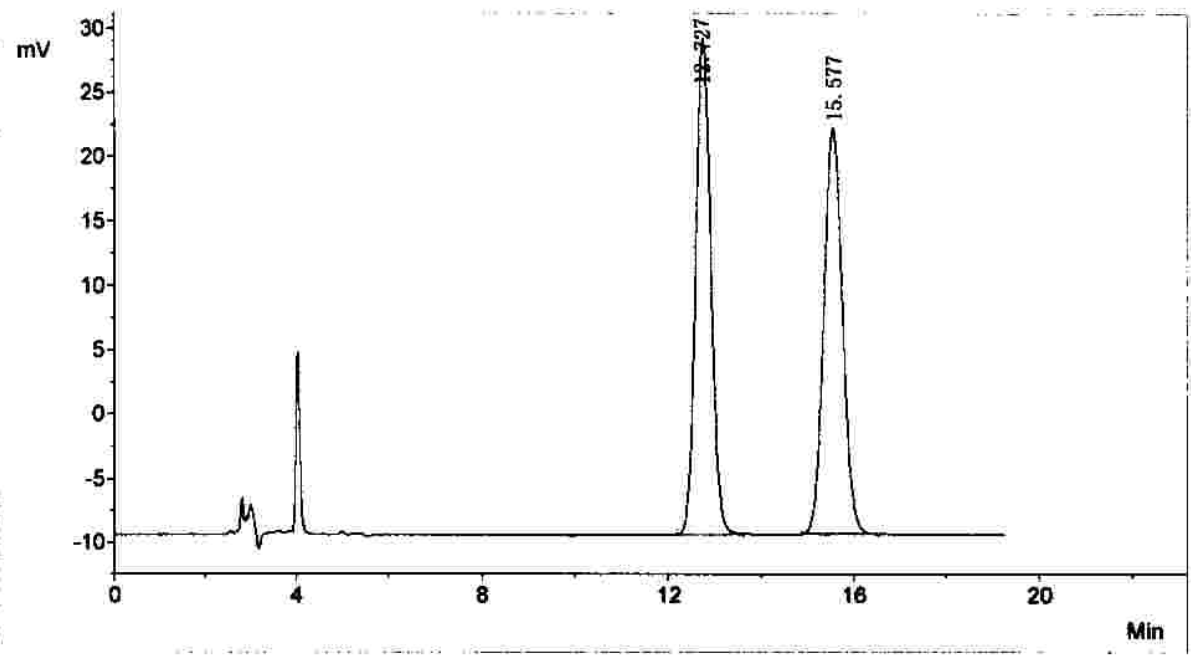

\begin{tabular}{ccccccc}
\multicolumn{2}{c}{ No. PeakNo } & ID. Name & R. Time & PeakHeight & PeakArea & PerCent \\
1 & 1 & Unknown & 12.727 & 38246.2 & 881875.6 & 49.8984 \\
2 & 2 & Unknown & 15.577 & 31354.0 & 885468.0 & 50.1016 \\
& & & & 69600.1 & 1767343.6 & 100.0000
\end{tabular}

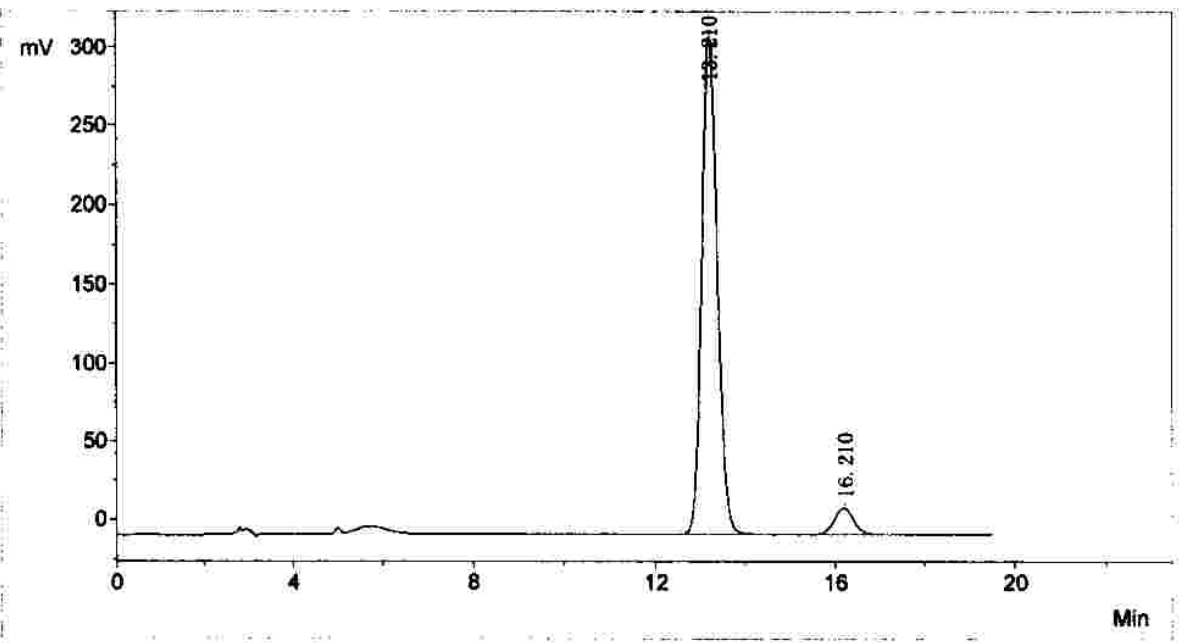

\begin{tabular}{|ccccrcr}
\multicolumn{2}{r}{ No. PeakNo } & ID. Name & R. Time & PeakHeight & \multicolumn{1}{c}{ PeakArea } & \multicolumn{1}{c}{ PerCent } \\
\hline 1 & 1 & Unknown & 13.210 & 315173.3 & 7588762.3 & 94.1286 \\
2 & 2 & Unknown & 16.210 & 16234.4 & 473355.9 & 5.8714 \\
Total & & & & 331407.7 & 8062118.2 & 100.0000
\end{tabular}



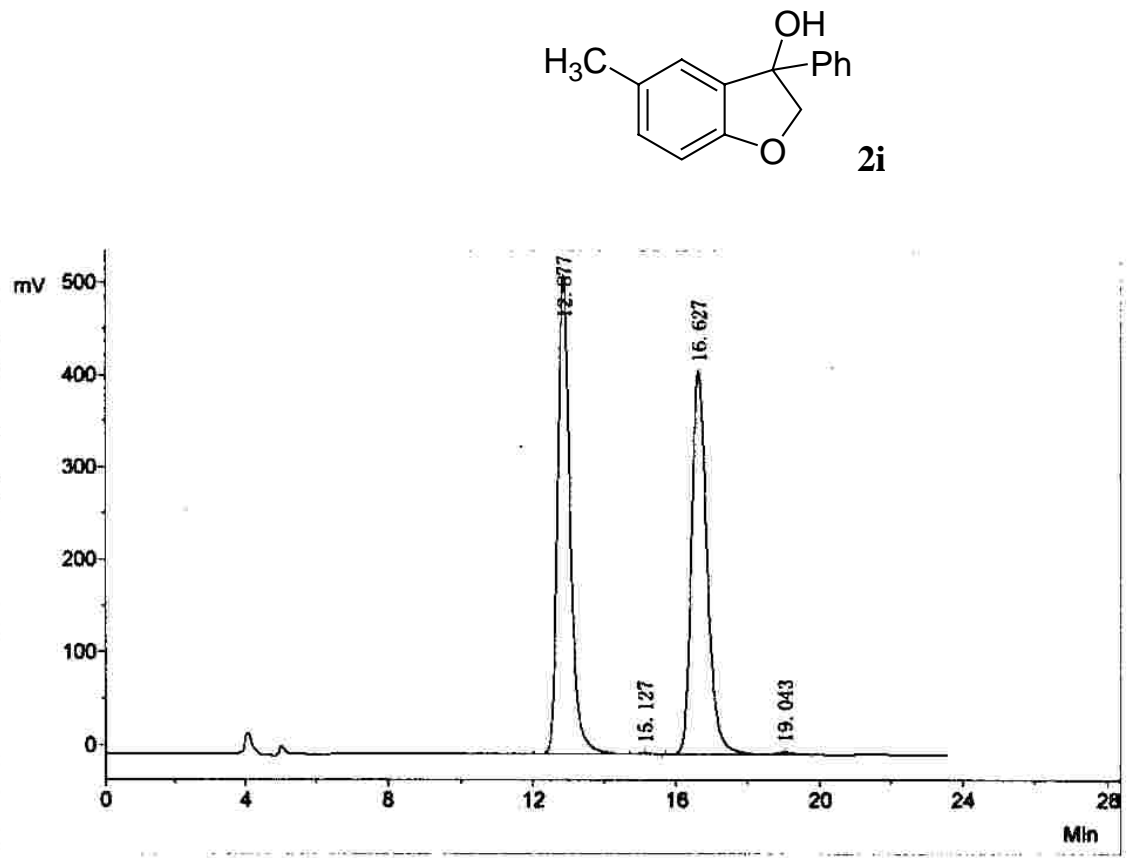

\begin{tabular}{ccccrrr}
\multicolumn{2}{c}{ No. PeakNo } & ID. Name & R. Time & PeakHeight & \multicolumn{1}{c}{ PeakArea } & \multicolumn{1}{c}{ PerCent } \\
1 & 1 & Unknown & 12.877 & 505745.4 & 12477102.9 & 49.8267 \\
2 & 2 & Unknown & 15.127 & 1349.9 & 31360.2 & 0.1252 \\
3 & 3 & Unknown & 16.627 & 413195.3 & 12463801.7 & 49.7736 \\
4 & 4 & Unknown & 19.043 & 2054.4 & 68740.2 & 0.2745 \\
\hline & & & & 922345.1 & 25041005.0 & 100.0000
\end{tabular}

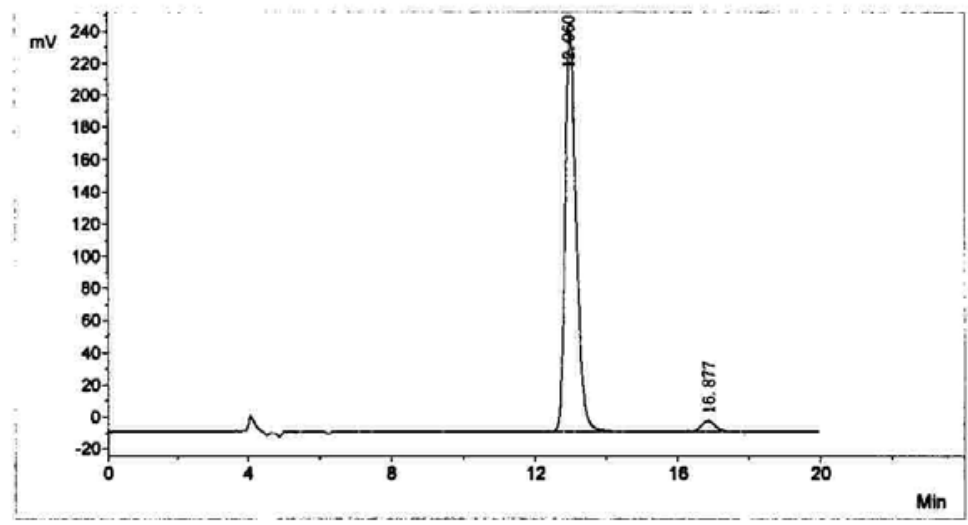

\begin{tabular}{rrrrrrr}
\multicolumn{2}{r}{ No. PeakNo } & ID. Name & R. Time & PeakHeight & \multicolumn{1}{c}{ PeakArea } & PerCent \\
\hline 1 & 1 & Unknown & 12.960 & 238169.9 & 5427120.7 & 96.7144 \\
2 & 2 & Unknown & 16.877 & 6571.9 & 184373.4 & 3.2856 \\
\hline
\end{tabular}


<smiles>OC1(c2ccccc2)CCc2ccccc21</smiles>

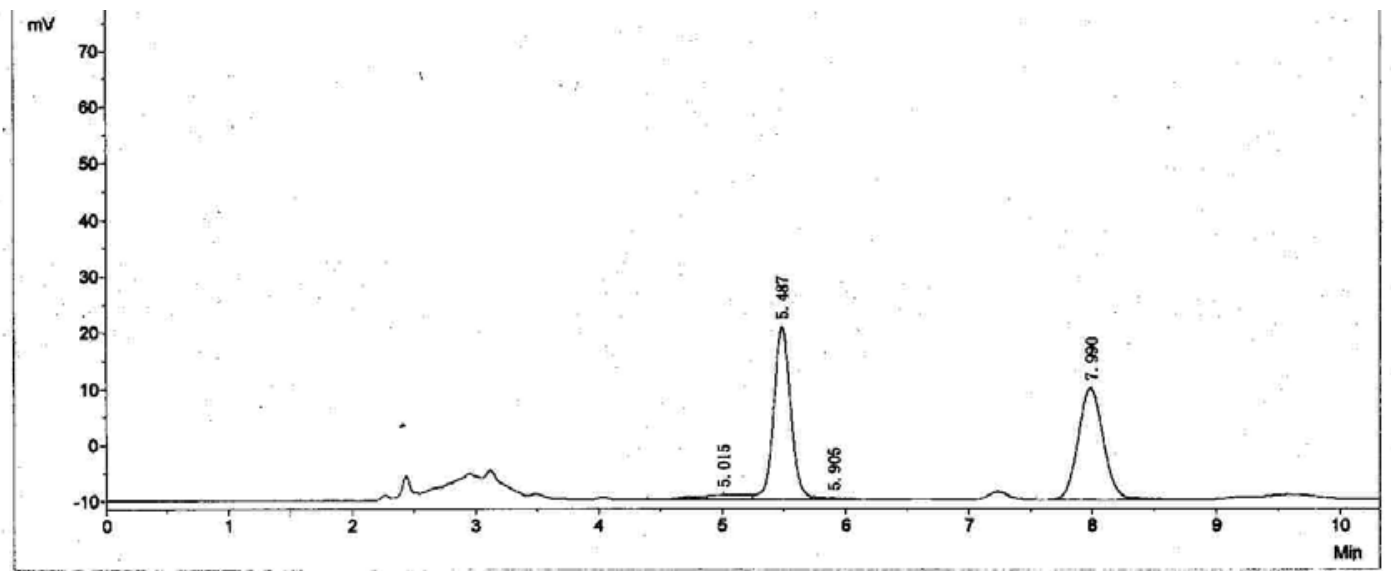

\begin{tabular}{rrrrr} 
No. & R. Time & PeakHeight & \multicolumn{1}{c}{ PeakArea } & \multicolumn{1}{c}{ PerCont } \\
\hline 1 & 5.015 & 791.6 & 24346.6 & 4.0391 \\
2 & 5.487 & 30603.6 & 293108.5 & 48.6269 \\
3 & 5.905 & 216.3 & 5229.2 & 0.8675 \\
4 & 7.990 & 19707.0 & 280086.2 & 46.4665 \\
\hline
\end{tabular}

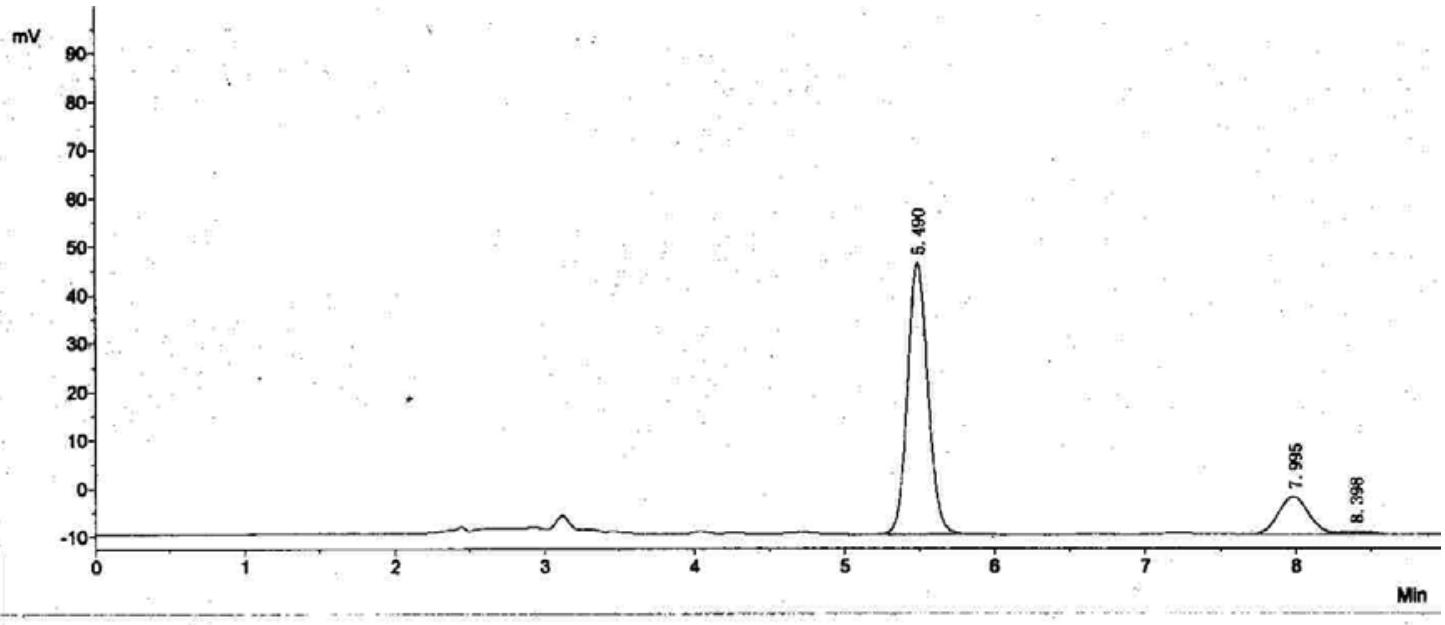

\begin{tabular}{rrrrr} 
No. & R. Time & PeakHeight & \multicolumn{1}{c}{ PeakArea } & \multicolumn{1}{c}{ PerGent } \\
\hline 1 & 5.490 & 55889.5 & 529952.5 & 82.1266 \\
2 & 7.995 & 7618.1 & 108076.5 & 16.7486 \\
3 & 8.398 & 519.6 & 7258.0 & 1.1248 \\
\hline Total & 64027.2 & 645287.0 & 100.0000
\end{tabular}



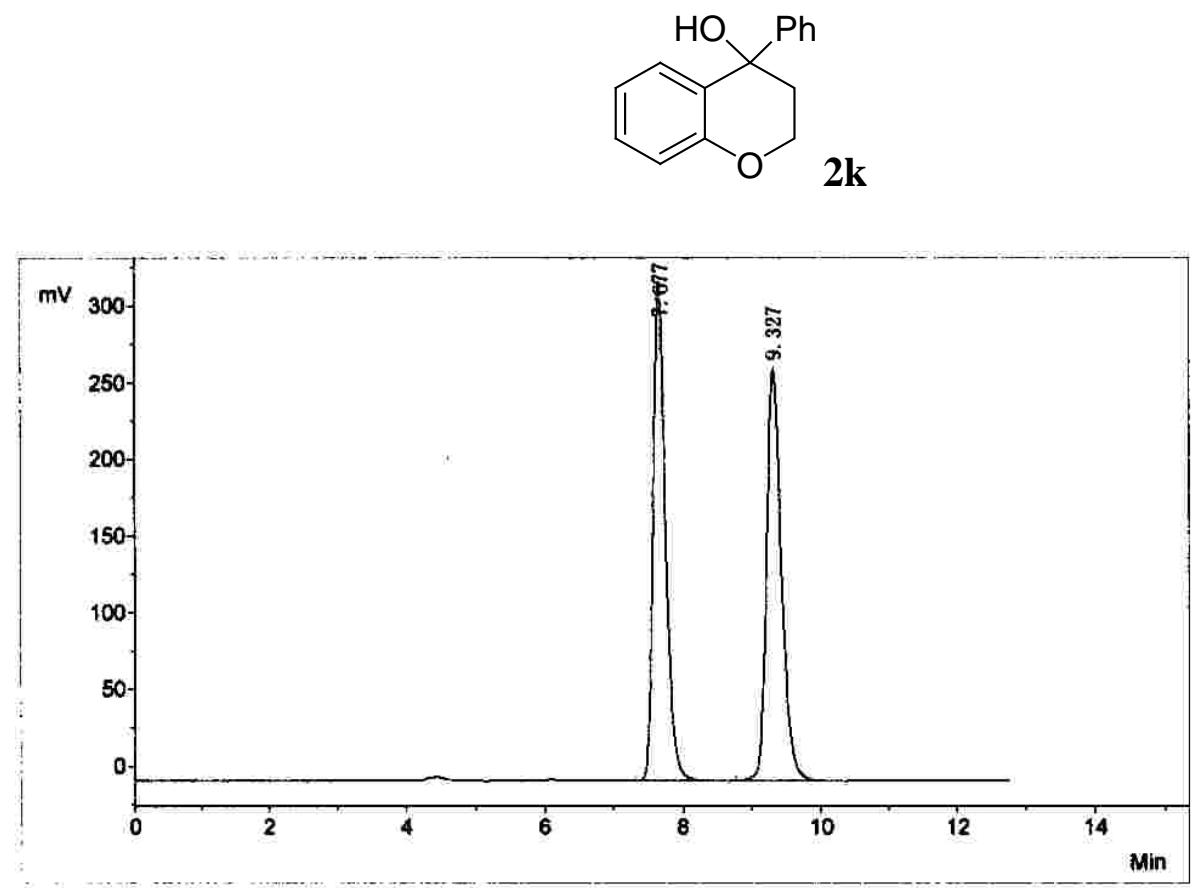

\begin{tabular}{ccccrcc}
\multicolumn{2}{c}{ No. PeakNo } & ID. Name & R. Time & PeakHeight & PeakArea & PerCent \\
\hline 1 & 1 & Unknown & 7.677 & 312496.9 & 4017364.1 & 49.8267 \\
2 & 2 & Unknown & 9.327 & 267421.9 & 4045317.0 & 50.1733 \\
\hline Total & & & 579918.8 & 8062681.1 & 100.0000
\end{tabular}

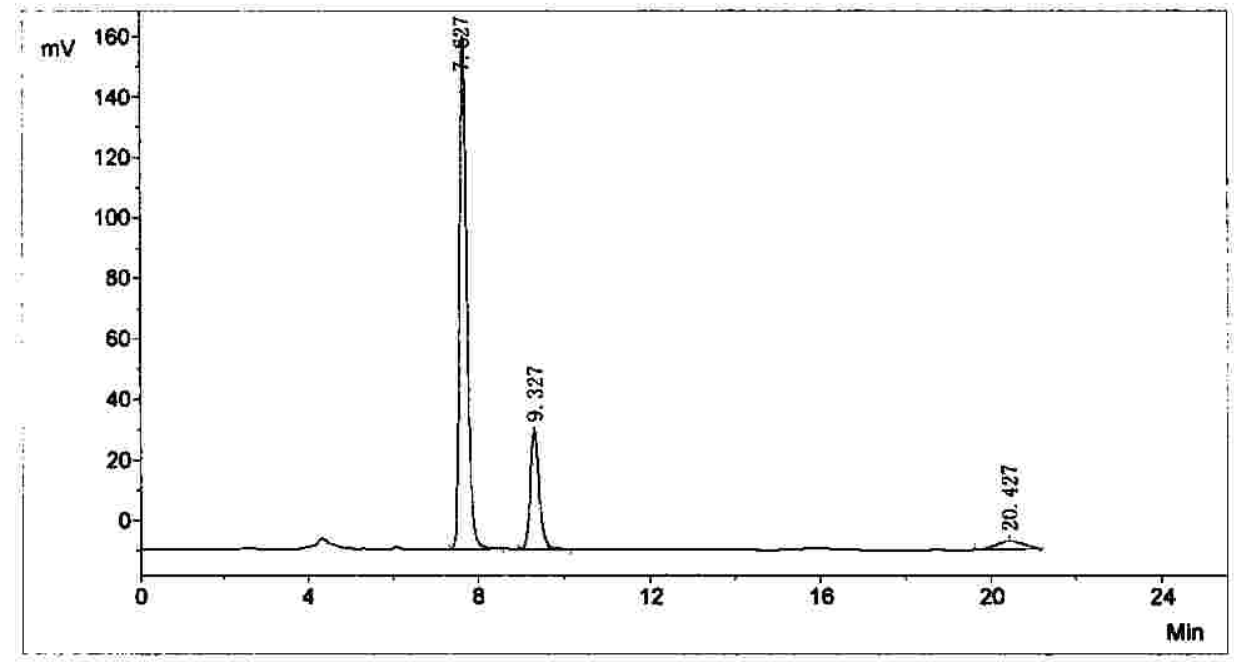

\begin{tabular}{rrrrrrr}
\multicolumn{1}{r}{ No. PeakNo } & ID. Name & R. Time & PeakHeight & \multicolumn{1}{c}{ PeakArea } & \multicolumn{1}{c}{ PerCent } \\
\hline 1 & 1 & Unknown & 7.627 & 164493.2 & 1999083.9 & 74.4370 \\
2 & 2 & Unknown & 9.327 & 38661.6 & 570540.2 & 21.2444 \\
3 & 3 & Unknown & 20.427 & 2604.9 & 115981.5 & 4.3186 \\
\hline Total & & & & 205759.6 & 2685605.6 & 100.0000
\end{tabular}

